\title{
Stochastic Viscoelastic Wave Equations with Nonlinear Damping and Source Terms
}

\author{
Shuilin Cheng, Yantao Guo, and Yanbin Tang \\ School of Mathematics and Statistics, Huazhong University of Science and Technology, Wuhan, Hubei 430074, China \\ Correspondence should be addressed to Yanbin Tang; tangybhust@sina.com
}

Received 23 December 2013; Revised 11 February 2014; Accepted 11 February 2014; Published 27 March 2014

Academic Editor: Francisco J. Marcellán

Copyright (C) 2014 Shuilin Cheng et al. This is an open access article distributed under the Creative Commons Attribution License, which permits unrestricted use, distribution, and reproduction in any medium, provided the original work is properly cited.

\begin{abstract}
The goal of this paper is to study an initial boundary value problem of stochastic viscoelastic wave equation with nonlinear damping and source terms. Under certain conditions on the initial data: the relaxation function, the indices of nonlinear damping, and source terms and the random force, we prove the local existence and uniqueness of solution by the Galerkin approximation method. Then, considering the relationship between the indices of nonlinear damping and nonlinear source, we give the necessary conditions of global existence and explosion in finite time in some sense of solutions, respectively.
\end{abstract}

\section{Introduction}

We consider a stochastic viscoelastic wave equation with nonlinear damping and source terms

$$
\begin{gathered}
u_{t t}-\Delta u+\int_{0}^{t} h(t-\tau) \Delta u(\tau) d \tau+\left|u_{t}\right|^{q-2} u_{t} \\
=|u|^{p-2} u+\varepsilon \sigma(x, t) \partial_{t} W(t, x), \\
u(x, t)=0, \quad(x, t) \in \partial D \times[0, T], \\
u(x, 0)=u_{0}(x), \quad u_{t}(x, 0)=u_{1}(x), \quad x \in \bar{D},
\end{gathered}
$$

where $D$ is a bounded domain in $R^{n}$ with smooth boundary $\partial D, q \geq 2, p \geq 2, \varepsilon$ is a given positive constant which measures the strength of noise, and $W(t, x)$ is an infinite dimensional Wiener process, $\sigma(x, t, \omega)$ is $L^{2}(D)$-valued progressively measurable and $h$ is a positive relaxation function satisfying some conditions to be specified later. By simplicity, we have set equal to 1 all the coefficients in the equation different from the random force.
For the deterministic case on viscoelastic wave equation, many authors studied the following problem:

$$
\begin{gathered}
u_{t t}-\Delta u+a\left|u_{t}\right|^{q-2} u_{t}=b|u|^{p-2} u, \quad(x, t) \in D \times(0, T), \\
u(x, t)=0, \quad(x, t) \in \partial D \times(0, T), \\
u(x, 0)=u_{0}(x), \quad u_{t}(x, 0)=u_{1}(x), \quad x \in \bar{D},
\end{gathered}
$$

where $a, b \geq 0$. If $a>0, b=0$. Haraux and Zuazua [1] and Kopáčková [2] proved that the damping term assures existence of global solution and decay of solution for arbitrary initial data. If $a=0, b>0$, Ball [3] and Kalantarov and Ladyzhenskaya [4] gave that the source term causes finite time blow-up with the large initial data. If $a>0, b>0$, the interaction between damping term and source term occurs; Levine et al. [5, 6] studied the linear damping (i.e., $q=2$ ) and proved that the solution with negative initial energy blows up in finite time; Georgiev and Todorova [7] considered nonlinear damping and source terms; they showed that the solution blows up in finite time if $p>q>2$ for sufficiently large initial data and exists globally if $q \geq p>2$ with large initial data. Alves et al. [8] and Rammaha [9] focused the nonlinear wave equations or systems on the influence between damping and source and described the existence, uniform decay rates, and blow-up to the solutions. 
In fact, lots of investigators have paid attention to the viscoelastic wave equation, which has its origin in the mathematical description of viscoelastic materials. The dynamic properties of viscoelastic materials are of great importance as they appear in many applications to natural sciences. The general viscoelastic wave equation has the following form:

$$
\begin{gathered}
u_{t t}-\Delta u+\int_{0}^{t} h(t-\tau) \Delta u(\tau) d \tau+g\left(u_{t}\right)=f(u), \\
(x, t) \in D \times(0, T), \\
u(x, t)=0, \quad(x, t) \in \partial D \times(0, T), \\
u(x, 0)=u_{0}(x), \quad u_{t}(x, 0)=u_{1}(x), \quad x \in \bar{D},
\end{gathered}
$$

where $h$ is a relaxation function and $f, g$ are given functions. If $f$ is nonlinear, $g$ is linear; Kafini and Messaoudi [10] established a blow-up result; if $f(u)=|u|^{p} u, g\left(u_{t}\right)=\left|u_{t}\right|^{q} u_{t}$. Messaoudi [11] showed that the solution with negative initial energy blows up in finite time if $p>q$ and exists globally if $q \geq p$ under suitable conditions on relaxation function $h$. This blow-up result has been pushed to the case of positive initial energy by Messaoudi [12]. For $g(w)=-\Delta w$, Song and Zhong [13] obtained that the solution with positive initial energy blows up in finite time. Ikehata [14] gave some remarks on the wave equations with nonlinear damping and source terms. Aassila et al. [15], Cavalcanti et al. [16], and Cavalcanti et al. [17] studied the boundary damping and proved the existence and uniform decay of the solutions. Cavalcanti et al. [18] discussed the asymptotic stability of the wave equation on a compact Riemannian manifold; they proved that the solutions of the corresponding partial viscoelastic model decay exponentially to zero under some conditions.

Under the consideration of random environment, some authors investigated the following stochastic wave equation with nonlinear damping and source terms:

$$
\begin{array}{r}
u_{t t}-\Delta u+g\left(u_{t}\right)=f(u)+\varepsilon \sigma(u, \nabla u, x, t) \partial_{t} W(t, x), \\
(x, t) \in D \times(0, T), \\
u(x, t)=0, \quad(x, t) \in \partial D \times(0, T), \\
u(x, 0)=u_{0}(x), \quad u_{t}(x, 0)=u_{1}(x), \quad x \in \bar{D},
\end{array}
$$

where $W(t, x)$ is a $V$-valued $R$-Wiener process on some completed probability space, and $R$ is a nonnegative operator with finite trace on $V$ (see [19-26]). If $f(w)=|w|^{p-2} w, g(w)=$ $-\Delta w$ or $w$; Bo et al. [27] showed that the solution blows up with positive probability or it is explosive in $L^{2}$ sense. If $g(w)=|w|^{q-2} w$, Gao et al. [28] showed that the global solution exists for $q>p$, and the solution blows up with positive probability or is explosive in energy sense for $p>q$.
Recently, Wei and Jiang [29] and Liang and Gao [30] considered the following nonlinear stochastic viscoelastic wave equation with linear damping:

$$
\begin{gathered}
u_{t t}-\Delta u+\int_{0}^{t} h(t-\tau) \Delta u(\tau) d \tau+u_{t} \\
=|u|^{p-2} u+\varepsilon \sigma(u, \nabla u, x, t) \partial_{t} W(t, x), \\
u(x, t)=0, \quad(x, t) \in \partial D \times(0, T), \\
u(x, 0)=u_{0}(x), \quad u_{t}(x, 0)=u_{1}(x), \quad x \in \bar{D},
\end{gathered}
$$

and they used the fixed point theorem to prove the existence and uniqueness of local mild solution; then by an appropriate energy inequality and estimations, they obtained the global existence and the decay estimate of the energy function of the solution and showed that the solution blows up with positive probability or it is explosive in $L^{2}$ sense under some conditions.

As we know, no one considers the stochastic viscoelastic wave equation (1) with the interaction between nonlinear damping and nonlinear source terms. In this paper, we study the global existence and the explosive phenomena under some suitable conditions on the nonlinear damping and nonlinear source terms.

In contrast with the model in [27], we add a viscoelastic term $\int_{0}^{t} h(t-\tau) \Delta u(\tau) d \tau$ and use the nonlinear damping term $\left|u_{t}\right|^{q-2} u_{t}$ instead of the linear damping $k u_{t}$ and the strong damping $\Delta u_{t}$. To the model in [28], we add a viscoelastic term $\int_{0}^{t} h(t-\tau) \Delta u(\tau) d \tau$. To the model in $[29,30]$, we use the nonlinear damping term $\left|u_{t}\right|^{q-2} u_{t}$ instead of the linear damping $u_{t}$. To the model in [12], we add a random force. In this paper, we generalize the blow-up and global existence results to the solution of (1) with interaction among viscoelastic memory, nonlinear damping, nonlinear source, and random force.

This paper is organized as follows. In the next section, we recall some preliminaries related to assumptions and definitions for the solutions of the stochastic equations. In Section 3, we use the Galerkin approximation method to get the local solution of stochastic viscoelastic wave equations with nonlinear damping and source terms. In Section 4, by the energy function and some estimates, we prove that the solution blows up with positive probability or it is explosive in energy sense for $p>q$. In the last section, we obtain the existence of global solution by the Borel-Cantelli Lemma.

\section{Preliminaries}

Let $\left(X,\|\cdot\|_{X}\right)$ be a separable Hilbert space with Borel $\sigma$ algebra $\mathscr{B}(X)$, and let $(\Omega, \mathscr{F}, P)$ be a probability space. We set $H=L^{2}(D)$ with the inner product and norm denoted by $(\cdot, \cdot)$ and $\|\cdot\|_{2}$, respectively. Denote by $\|\cdot\|_{q}$ the $L^{q}(D)$ norm for $1 \leq q \leq \infty$ and by $\|\nabla \cdot\|_{2}$ the Dirichlet norm in $V=H_{0}^{1}(D)$ 
which is equivalent to $H^{1}(D)$ norm. We also assume that $q, p$ satisfy

$$
q \geq 2, \quad p>2, \quad \max \{p, q\} \leq \frac{2(n-1)}{n-2}, \quad \text { if } n \geq 3,
$$

$$
q \geq 2, \quad p>2, \quad \text { if } n=1,2 .
$$

Lemma 1 (see [27]). For all $u, v \in H^{1}\left(R^{n}\right)$ and $0<\rho \leq(2 /(n-$ 2)) $(n \geq 3)$ or $\rho>0(n=1,2)$, there exists a constant $c_{1}=$ $c_{1}(n, \rho)>0$ such that

$$
\|u\|_{L^{2(\rho+1)}} \leq c_{1}\|u\|_{H^{1}}, \quad\left\|u^{\rho} v\right\|_{L^{2}} \leq c_{1}^{\rho+1}\|u\|_{H^{1}}^{\rho}\|v\|_{H^{1}} .
$$

One assumes that $h: R^{+} \rightarrow R^{+}$is a bounded nonincreasing $C^{1}$ function satisfying $h(0)>0,1-\int_{0}^{\infty} h(s) d s=l>0$, and there exist positive constants $\xi_{1}$ and $\xi_{2}$ such that

$$
-\xi_{1} h(t) \leq h^{\prime}(t) \leq-\xi_{2} h(t), \quad t \geq 0 .
$$

In this paper, $E(\cdot)$ stands for expectation with respect to probability measure $P, W(t, x)(t \geq 0)$ is a $V$-valued $R$ Wiener process on the probability space with the covariance operator $R$ satisfying $\operatorname{Tr}(R)<\infty$. A complete orthonormal system $\left\{e_{k}\right\}_{k=1}^{\infty}$ in $V$ with $C_{0}:=\sup _{k \geq 1}\left\|e_{k}\right\|_{\infty}<\infty$, and a bounded sequence of nonnegative real numbers $\left\{\lambda_{k}\right\}_{k=1}^{\infty}$ satisfies that $\mathrm{Re}_{k}=\lambda_{k} e_{k}, k=1,2, \ldots$

To simplify the computations, we assume that the covariance operator $R$ and Laplacian $-\Delta$ with homogeneous Dirichlet boundary condition have a common set of eigenfunctions, that is,

$$
\begin{aligned}
-\Delta e_{k} & =\alpha_{k} e_{k}, \quad x \in D, \\
e_{k} & =0, \quad x \in \partial D,
\end{aligned}
$$

and then, for any $t \in[0, T], W(t, x)$ has an expansion

$$
W(t, x)=\sum_{k=1}^{\infty} \sqrt{\lambda_{k}} \beta_{k}(t) e_{k}(x),
$$

where $\left\{\beta_{k}(t)\right\}_{k=1}^{\infty}$ are real valued Brownian motions mutually independent on $(\Omega, \mathscr{F}, P)$. Let $\mathscr{H}$ be the set of $L_{2}^{0}=L^{2}\left(R^{1 / 2} V\right.$, $V$ )-valued processes with the norm

$$
\begin{aligned}
\|\Phi(t)\|_{\mathscr{H}} & =\left(E \int_{0}^{t}\|\Phi(s)\|_{L_{2}^{0}}^{2} d s\right)^{1 / 2} \\
& =\left(E \int_{0}^{t} \operatorname{Tr}\left(\Phi(s) R \Phi^{*}(s)\right) d s\right)^{1 / 2}<\infty
\end{aligned}
$$

where $\Phi^{*}(s)$ denotes the adjoint operator of $\Phi(s)$. For any process $\Phi(t) \in \mathscr{H}$, we can define the stochastic integral with respect to the $R$-Wiener process as $\int_{0}^{t} \Phi(s) d W(s)$, which is a martingale. For more details about the infinite dimension Wiener process and the stochastic integral, we refer the readers to [21].

Definition 2. Assume that $\left(u_{0}, u_{1}\right) \in H_{0}^{1}(D) \times L^{2}(D)$, and $E \int_{0}^{T}\|\sigma(t)\|_{2}^{2} d t<\infty ; u$ is said to be a solution of (1) on the interval $[0, T]$, if $\left(u, u_{t}\right)$ is $H_{0}^{1}(D) \times L^{2}(D)$-valued progressively measurable, $\left(u, u_{t}\right) \in L^{2}\left(\Omega ; C\left([0, T] ; H_{0}^{1}(D) \times L^{2}(D)\right)\right)$, $u_{t} \in L^{q}((0, T) \times D)$, and such that (1) holds in the sense of distributions over $(0, T) \times D$ for almost all $\omega$.

\section{Local Existence and Uniqueness}

In this section, we establish the local existence and uniqueness of solution to problem (1) by the Galerkin approximation method. Set $f(u)=|u|^{p-2} u, g(s)=|s|^{q-2}$ s. For each $N \geq 1$, we define a cut-off function $\chi_{N} \in C_{0}^{\infty}$, such that $0 \leq \chi_{N}(s) \leq$ $1,\left\|\chi_{N}^{\prime}(s)\right\|_{\infty} \leq 2$ for $s \in R$, and

$$
\chi_{N}(s)= \begin{cases}1, & |s| \leq N \\ 0, & |s| \geq N+1 .\end{cases}
$$

Denote $f_{N}(u)=\chi_{N}\left(\|\nabla u\|_{2}\right) f(u)$ for $u \in H_{0}^{1}(D)$, then, Lemma 1 implies that

$$
\left\|f_{N}(u)-f_{N}(v)\right\|_{2} \leq C_{N}\|u-v\|_{2}, \quad \text { for } u, v \in H_{0}^{1}(D),
$$

where $\mathrm{C}_{N}$ is a constant depending only on $N$.

For any $\lambda>0$, the Yosida approximation of mapping $g$ is

$$
g_{\lambda}(x)=\frac{1}{\lambda}\left(x-(I+\lambda g)^{-1}(x)\right)=g(I+\lambda g)^{-1}(x), \quad x \in R,
$$

and it has the following properties (see $[28,31,32]$ ):

$$
\begin{gathered}
g_{\lambda}(x) \in C^{1}(R), \quad 0 \leq g_{\lambda}^{\prime}(x) \leq \frac{1}{\lambda}, \\
\left|g_{\lambda}(x)\right| \leq|g(x)|, \quad\left|g_{\lambda}(x)\right| \leq \frac{1}{\lambda}|x|, \quad \forall x \in R .
\end{gathered}
$$

Lemma 3 (see [28]). Let $\left\{\lambda_{n}\right\}$ be a sequence of positive numbers, and let $\left\{x_{n}\right\}$ be a sequence of real numbers such that $\lambda_{n} \rightarrow 0$ and $x_{n} \rightarrow x$ as $n \rightarrow \infty$, then

$$
\lim _{n \rightarrow \infty} g_{\lambda_{n}}\left(x_{n}\right)=g(x) .
$$

Lemma 4 (see [33]). Let $D$ be a bounded domain in $R^{n}(n \geq$ 1). Suppose that $\left\{\phi_{k}\right\}$ is a bounded sequence in $L^{q}(D)(1<q<$ $\infty)$, such that $\phi_{k}(x) \rightarrow \phi(x)$ for almost all $x \in D$, for some $\phi(x) \in L^{q}(D)$. Then $\phi_{k}(x) \rightarrow \phi(x)$ weakly in $L^{q}(D)$.

Fix $\lambda>0$ and $N>0$; we consider the regularized initial boundary value problem

$$
\begin{gathered}
u_{t t}-\Delta u+\int_{0}^{t} h(t-\tau) \Delta u(\tau) d \tau+g_{\lambda}\left(u_{t}\right) \\
=f_{N}(u)+\varepsilon \sigma(x, t) \partial_{t} W(t, x), \\
u(x, t)=0, \quad(x, t) \in \partial D \times(0, T), \\
u(x, 0)=u_{0}(x), \quad u_{t}(x, 0)=u_{1}(x), \quad x \in \bar{D},
\end{gathered}
$$

with the initial data

$$
\left(u_{0}, u_{1}\right) \in\left(H^{2}(D) \cap H_{0}^{1}(D)\right) \times H_{0}^{1}(D),
$$


and $\sigma(x, t)$ is $H_{0}^{1}(D) \cap L^{\infty}(D)$-valued progressively measurable such that

$$
E \int_{0}^{T}\left(\|\nabla \sigma(t)\|_{2}^{2}+\|\sigma(t)\|_{\infty}^{2}\right) d t<\infty
$$

For notational convenience, we omit $D$ in the Hilbert space.

Lemma 5. Assume (18), (19), and the conditions on $h$ hold. Then there is a pathwise unique solution $u$ of (17) such that $u \in$ $L^{2}\left(\Omega ; L^{\infty}\left(0, T ; H^{2} \cap H_{0}^{1}\right)\right) \cap L^{2}\left(\Omega ; C\left([0, T] ; H_{0}^{1}\right)\right)$, and $u_{t} \in$ $L^{2}\left(\Omega ; L^{\infty}\left(0, T ; H_{0}^{1}\right)\right) \cap L^{2}\left(\Omega ; C\left([0, T] ; L^{2}\right)\right)$. Moreover, it holds that

$$
\begin{array}{r}
E\left(\left\|u_{t}\right\|_{L^{\infty}\left(0, T ; H_{0}^{1}\right)}^{2}+\|u\|_{L^{\infty}\left(0, T ; H^{2} \cap H_{0}^{1}\right)}^{2}\right. \\
\left.+\int_{0}^{T} \int_{D} g_{\lambda}\left(u_{t}\right) u_{t} d x d t\right) \leq C,
\end{array}
$$

where $C$ denotes a positive constant independent of $\lambda$.

Proof. Let $u_{m}(x, t)=\sum_{j=1}^{m} a_{m, j} e_{j}(x)$ and let $a_{m, j}(j=1,2, \ldots$, $m)$ be the solution of the following system:

$$
\begin{aligned}
& \left(u_{m}^{\prime \prime}(t), e_{j}(x)\right)+\left(\nabla u_{m}, e_{j}\right) \\
& \quad-\int_{0}^{t} h(t-\tau)\left(\nabla u_{m}(\tau), \nabla e_{j}\right) d \tau+\left(g_{\lambda}\left(u_{m}^{\prime}\right), e_{j}\right) \\
& =\left(f_{N}\left(u_{m}\right), e_{j}\right)+\varepsilon\left(e_{j}, \sigma d W_{t}\right), \quad(x, t) \in D \times(0, T), \\
& u_{m}(0)=\sum_{j=1}^{m}\left(u_{0}, e_{j}\right), \quad u_{m}^{\prime}(0)=\sum_{j=1}^{m}\left(u_{1}, e_{j}\right), \quad x \in D .
\end{aligned}
$$

By Itô formula, we have

$$
\begin{aligned}
& \left\|u_{m}^{\prime}(t)\right\|_{2}^{2}+\left\|\nabla u_{m}(t)\right\|_{2}^{2} \leq\left\|u_{m}^{\prime}(0)\right\|_{2}^{2}+\left\|\nabla u_{m}(0)\right\|_{2}^{2} \\
& \quad-2 \int_{0}^{t} \int_{D} \int_{0}^{s} h(s-\tau)\left(\Delta u_{m}(\tau)\right) d \tau u_{m}^{\prime}(s) d x d s \\
& \quad+2 \int_{0}^{t} \int_{D} f_{N}\left(u_{m}\right) u_{m}^{\prime}(s) d x d s+2 \varepsilon \int_{0}^{t}\left(u_{m}^{\prime}(s), \sigma d \mathrm{~W}_{s}\right) \\
& \quad-2 \int_{0}^{t} \int_{D} g_{\lambda}\left(u_{m}^{\prime}\right) u_{m}^{\prime}(s) d x d s \\
& \quad+\operatorname{Tr}(R) C_{0}^{2} \sum_{j=1}^{\infty} \int_{0}^{t}\left|\left(e_{j}, \varepsilon \sigma\right)\right|^{2} d s,
\end{aligned}
$$

$$
\begin{aligned}
& \left\|\nabla u_{m}^{\prime}(t)\right\|_{2}^{2}+\left\|\Delta u_{m}(t)\right\|_{2}^{2} \leq\left\|\nabla u_{m}^{\prime}(0)\right\|_{2}^{2}+\left\|\Delta u_{m}(0)\right\|_{2}^{2} \\
& -2 \int_{0}^{t} \int_{D} \int_{0}^{s} h(s-\tau) \Delta u_{m}(\tau) d \tau \Delta u_{m}^{\prime}(s) d x d s \\
& -2 \int_{0}^{t} \int_{D} f_{N}\left(u_{m}\right) \Delta u_{m}^{\prime}(s) d x d s \\
& +2 \varepsilon \int_{0}^{t}\left(\nabla u_{m}^{\prime}(s), \nabla\left(\sigma d W_{s}\right)\right) \\
& +2 \int_{0}^{t} \int_{D} g_{\lambda}\left(u_{m}^{\prime}\right) \Delta u_{m}^{\prime}(s) d x d s \\
& +\operatorname{Tr}(R) C_{0}^{2} \sum_{j=1}^{\infty} \int_{0}^{t}\left|\left(e_{j}, \varepsilon \nabla \sigma\right)\right|^{2} d s \\
& +\sum_{j=1}^{\infty} \sum_{i=1}^{\infty} \lambda_{i} \int_{0}^{t}\left|\left(e_{j}, \varepsilon \sigma \nabla e_{i}\right)\right|^{2} d s,
\end{aligned}
$$

for all $t \in[0, T]$ and almost all $\omega$, where

$$
\operatorname{Tr}(R)=\sum_{i=1}^{\infty} \lambda_{i}<\infty, \quad C_{0}=\sup _{k \geq 1}\left\|e_{k}\right\|_{\infty}<\infty .
$$

Using Hölder's inequality, Young's inequality, Poincaré's inequality, and Lemma 1, we have

$$
\begin{aligned}
-2 \int_{D} f_{N}\left(u_{m}\right) u_{m}^{\prime}(s) d x & \leq 2\left\|f_{N}\left(u_{m}\right)\right\|_{2}\left\|u_{m}^{\prime}\right\|_{2} \\
& \leq 2 C_{N}\left\|u_{m}\right\|\left\|u_{m}^{\prime}\right\|_{2} \\
& \leq C\left\|\nabla u_{m}\right\|_{2}\left\|u_{m}^{\prime}\right\|_{2} \\
& \leq C\left\|\nabla u_{m}\right\|_{2}^{2}+C\left\|u_{m}^{\prime}\right\|_{2}^{2},
\end{aligned}
$$

and so we have

$$
\begin{aligned}
& -2 \int_{0}^{t} \int_{D} f_{N}\left(u_{m}\right) u_{m}^{\prime}(s) d x d s \\
& \quad \leq C \int_{0}^{t}\left\|\nabla u_{m}\right\|_{2}^{2} d s+C \int_{0}^{t}\left\|u_{m}^{\prime}\right\|_{2}^{2} d s .
\end{aligned}
$$

Since

$$
\begin{aligned}
- & 2 \int_{D} f_{N}\left(u_{m}\right) \Delta u_{m}^{\prime}(s) d x \\
& =2(p-1) \int_{D} \chi_{N}\left(\left\|\nabla u_{m}\right\|_{2}\right)\left|u_{m}\right|^{p-2} \nabla u_{m} \nabla u_{m}^{\prime} d x \\
& \leq 2(p-1)\left\|\nabla u_{m}^{\prime}\right\|_{2}\left\|\chi_{N}\left(\left\|\nabla u_{m}\right\|_{2}\right)\left|u_{m}\right|^{p-2} \nabla u_{m}\right\|_{2} \\
& \leq C(p-1)\left\|\nabla u_{m}^{\prime}\right\|_{2}\left\|\nabla u_{m}\right\|_{H^{1}} \chi_{N}\left(\left\|\nabla u_{m}\right\|_{2}\right)\left\|u_{m}\right\|_{H^{1}}^{p-2} \\
& \leq C(p-1)\left\|\nabla u_{m}^{\prime}\right\|_{2}\left\|\Delta u_{m}\right\|_{2} \chi_{N}\left(\left\|\nabla u_{m}\right\|_{2}\right)\left\|\nabla u_{m}\right\|_{2}^{p-2} \\
& \leq C(p-1)(N+1)^{p-2}\left\|\nabla u_{m}^{\prime}\right\|_{2}\left\|\Delta u_{m}\right\|_{2} \\
& \leq C\left\|\Delta u_{m}\right\|_{2}^{2}+C\left\|\nabla u_{m}^{\prime}\right\|_{2}^{2},
\end{aligned}
$$


thus we have

$$
\begin{aligned}
& -2 \int_{0}^{t} \int_{D} f_{N}\left(u_{m}\right) \Delta u_{m}^{\prime}(s) d x d s \\
& \quad \leq C \int_{0}^{t}\left\|\Delta u_{m}\right\|_{2}^{2} d s+C \int_{0}^{t}\left\|\nabla u_{m}^{\prime}\right\|_{2}^{2} d s
\end{aligned}
$$

where $C$ depends on the fixed number $N$.

Due to integration by parts, we have

$$
\int_{D} g_{\lambda}\left(u_{m}^{\prime}\right) \Delta u_{m}^{\prime} d x=-\int_{D} g_{\lambda}^{\prime}\left(u_{m}^{\prime}\right)\left|\nabla u_{m}^{\prime}\right|^{2} d x<0
$$

Since $g_{\lambda}(x)$ is the Yosida approximation of mapping $g(x)$, by the Lemma 3 and sign-preserving theorem of limit, for small enough $\lambda$, we have

$$
\left(g_{\lambda}\left(u_{m}^{\prime}(t)\right), u_{m}^{\prime}(t)\right) \geq 0
$$

Moreover, by the conditions of $h$, we get

$$
\begin{aligned}
& \int_{0}^{t} \int_{D} h(t-\tau) \Delta u_{m}(\tau) u_{m}^{\prime}(t) d x d \tau \\
& =-\frac{d}{d t} \int_{0}^{t} h(t-\tau)\left(\nabla u_{m}(\tau), \nabla u_{m}(t)\right) d \tau \\
& +h(0)\left(\nabla u_{m}(t), \nabla u_{m}(t)\right) \\
& +\int_{0}^{t} h^{\prime}(t-\tau)\left(\nabla u_{m}(\tau), \nabla u_{m}(t)\right) d \tau, \\
& -2 \int_{0}^{t} \int_{0}^{s}\left(h(s-\tau) \Delta u_{m}(\tau), u_{m}^{\prime}(s)\right) d \tau d s \\
& =2 \int_{0}^{t} h(t-\tau)\left(\nabla u_{m}(\tau), \nabla u_{m}(t)\right) d \tau \\
& -2 h(0) \int_{0}^{t}\left(\nabla u_{m}(s), \nabla u_{m}(s)\right) d s \\
& -2 \int_{0}^{t} \int_{0}^{s} h^{\prime}(s-\tau)\left(\nabla u_{m}(\tau), \nabla u_{m}(s)\right) d \tau d s, \\
& 2 \int_{0}^{t} \int_{0}^{s}\left(h(s-\tau) \Delta u_{m}(\tau), \Delta u_{m}^{\prime}(s)\right) d \tau d s \\
& =2 \int_{0}^{t} h(t-\tau)\left(\Delta u_{m}(\tau), \Delta u_{m}(t)\right) d \tau \\
& -2 h(0) \int_{0}^{t}\left(\Delta u_{m}(s), \Delta u_{m}(s)\right) d s \\
& -2 \int_{0}^{t} \int_{0}^{s} h^{\prime}(s-\tau)\left(\Delta u_{m}(\tau), \Delta u_{m}(s)\right) d \tau d s .
\end{aligned}
$$

Next, from the properties of $h$, we have

$$
\begin{aligned}
& 2 \int_{0}^{t} h(t-\tau)\left(\nabla u_{m}(\tau), \nabla u_{m}(t)\right) d \tau \\
& \leq \frac{1}{2}\left\|\nabla u_{m}(t)\right\|_{2}^{2}+C\left\|\int_{0}^{t} h(t-\tau) \nabla u_{m}(\tau) d \tau\right\|_{2}^{2} \\
& \leq \frac{1}{2}\left\|\nabla u_{m}(t)\right\|_{2}^{2}+C \int_{0}^{t}\left\|\nabla u_{m}(\tau)\right\|_{2}^{2} d \tau \\
& -2 h(0) \int_{0}^{t}\left(\nabla u_{m}(s), \nabla u_{m}(s)\right) d s \\
& =-2 h(0) \int_{0}^{t}\left\|\nabla u_{m}(s)\right\|_{2}^{2} d s \leq 0, \\
& \left|-2 \int_{0}^{t} \int_{0}^{s} h^{\prime}(s-\tau)\left(\nabla u_{m}(\tau), \nabla u_{m}(s)\right) d \tau d s\right| \\
& \leq 2 \int_{0}^{t} \int_{0}^{s}\left|h^{\prime}(s-\tau)\right|\left\|\nabla u_{m}(\tau)\right\|_{2}\left\|\nabla u_{m}(s)\right\|_{2} d \tau d s \\
& \leq C \int_{0}^{t} \int_{0}^{s} h(s-\tau)\left\|\nabla u_{m}(\tau)\right\|_{2}\left\|\nabla u_{m}(s)\right\|_{2} d \tau d s \\
& \leq \operatorname{Ch}(0) \int_{0}^{t} \int_{0}^{s}\left\|\nabla u_{m}(\tau)\right\|_{2} d \tau\left\|\nabla u_{m}(s)\right\|_{2} d s \\
& \leq \operatorname{Ch}(0)\left(\int_{0}^{t}\left\|\nabla u_{m}(\tau)\right\|_{2} d \tau\right)^{2} \\
& \leq \operatorname{Ch}(0) T \int_{0}^{t}\left\|\nabla u_{m}(\tau)\right\|_{2}^{2} d \tau \leq C \int_{0}^{t}\left\|\nabla u_{m}(\tau)\right\|_{2}^{2} d \tau, \\
& \left|-2 \int_{0}^{t} h(t-\tau)\left(\Delta u_{m}(\tau), \Delta u_{m}(t)\right) d \tau\right| \\
& \leq \frac{1}{2}\left\|\Delta u_{m}(t)\right\|_{2}^{2}+C \int_{0}^{t}\left\|\Delta u_{m}(\tau)\right\|_{2}^{2} d \tau,
\end{aligned}
$$

and similar to the derivation of (34), we have

$$
\begin{aligned}
& 2 \int_{0}^{t} \int_{0}^{s} h^{\prime}(s-\tau)\left(\Delta u_{m}(\tau), \Delta u_{m}(s)\right) d \tau d s \\
& \quad \leq C \int_{0}^{t}\left\|\Delta u_{m}(\tau)\right\|_{2}^{2} d \tau .
\end{aligned}
$$

By the B-D-G inequality and Young's inequality, we have

$$
\begin{aligned}
E\left(\sup _{t \in[0, T]}\left|\int_{0}^{t}\left(u_{m}^{\prime}(s), \varepsilon \sigma d W_{s}\right)\right|\right) \\
\leq \varepsilon C E\left(\sup _{t \in[0, T]}\left\|u_{m}^{\prime}(t)\right\|_{2}\right. \\
\left.\times\left[\sum_{i=1}^{\infty} \int_{0}^{T}\left(\sigma(x, t) \operatorname{Re}_{i}, \sigma(x, t) e_{i}\right) d t\right]^{1 / 2}\right)
\end{aligned}
$$




$$
\begin{gathered}
\quad \leq \frac{1}{2} E \sup _{t \in[0, T]}\left\|u_{m}^{\prime}(t)\right\|_{2}^{2}+\varepsilon^{2} C E \int_{0}^{T}\|\sigma(t)\|_{2}^{2} d t, \\
E\left(\sup _{t \in[0, T]}\left|\int_{0}^{t}\left(\nabla u_{m}^{\prime}(s), \varepsilon \nabla\left(\sigma d W_{s}\right)\right)\right|\right) \\
\leq \frac{1}{2} E \sup _{t \in[0, T]}\left\|\nabla u_{m}^{\prime}(t)\right\|_{2}^{2} \\
+\varepsilon^{2} C E \int_{0}^{T}\left(\|\nabla \sigma(t)\|_{2}^{2}+\|\sigma(t)\|_{\infty}^{2}\right) d t .
\end{gathered}
$$

From (22)-(37), we get

$$
\begin{aligned}
E \sup _{t \in[0, T]}( & \left\|u_{m}^{\prime}(t)\right\|_{2}^{2}+\left\|\nabla u_{m}(t)\right\|_{2}^{2} \\
& \left.+\left\|\nabla u_{m}^{\prime}(t)\right\|_{2}^{2}+\left\|\Delta u_{m}(t)\right\|_{2}^{2}\right) \\
\leq & \left\|u_{m}^{\prime}(0)\right\|_{2}^{2}+\left\|\nabla u_{m}(0)\right\|_{2}^{2} \\
& +\left\|\nabla u_{m}^{\prime}(0)\right\|_{2}^{2}+\left\|\Delta u_{m}(0)\right\|_{2}^{2} \\
& -2 E \int_{0}^{T}\left(g_{\lambda}\left(u_{m}^{\prime}(s)\right), u_{m}^{\prime}(s)\right) d s \\
& +\frac{1}{2} E \sup _{t \in[0, T]}\left\|\nabla u_{m}\right\|_{2}^{2}+\frac{1}{2} E \sup _{t \in[0, T]}\left\|u_{m}^{\prime}\right\|_{2}^{2} \\
& +\frac{1}{2} E \sup _{t \in[0, T]}\left\|\Delta u_{m}\right\|_{2}^{2}+\frac{1}{2} E \sup _{t \in[0, T]}\left\|\nabla u_{m}^{\prime}\right\|_{2}^{2} \\
& +3 C E \int_{0}^{T}\left(\left\|u_{m}^{\prime}(s)\right\|_{2}^{2}+\left\|\nabla u_{m}(s)\right\|_{2}^{2}\right. \\
& +\sum_{j=1 i=1}^{\infty} \sum_{i}^{\infty} \lambda_{i} \int_{0}^{t}\left|\left(e_{j}, \varepsilon \sigma \nabla e_{i}\right)\right|^{2} d s, \\
& +\varepsilon^{2} C E \int_{0}^{T}\left(\|\sigma(t)\|_{2}^{2}+\|\nabla \sigma(t)\|_{2}^{2}+\|\sigma(t)\|_{\infty}^{2}\right) d t \\
& +\operatorname{Tr}(R) C_{0}^{2} \sum_{j=1}^{\infty} \int_{0}^{t}\left|\left(e_{j}, \varepsilon \sigma\right)\right|^{2} d s \\
& +\operatorname{Tr}(R) C_{0}^{2} \sum_{j=1}^{\infty} \int_{0}^{t}\left|\left(e_{j}, \varepsilon \nabla \sigma\right)\right|^{2} d s \\
& \\
& \\
&
\end{aligned}
$$

then,

$$
\begin{aligned}
E \sup _{t \in[0, T]}\left(\left\|u_{m}^{\prime}(t)\right\|_{2}^{2}+\left\|\nabla u_{m}(t)\right\|_{2}^{2}\right. \\
\left.+\left\|\nabla u_{m}^{\prime}(t)\right\|_{2}^{2}+\left\|\Delta u_{m}(t)\right\|_{2}^{2}\right)
\end{aligned}
$$

$$
\begin{aligned}
& \leq 2\left(\left\|u_{m}^{\prime}(0)\right\|_{2}^{2}+\left\|\nabla u_{m}(0)\right\|_{2}^{2}\right. \\
& \left.+\left\|\nabla u_{m}^{\prime}(0)\right\|_{2}^{2}+\left\|\Delta u_{m}(0)\right\|_{2}^{2}\right) \\
& -4 E \int_{0}^{T}\left(g_{\lambda}\left(u_{m}^{\prime}(s)\right), u_{m}^{\prime}(s)\right) d s \\
& +6 C E \int_{0}^{T}\left(\left\|u_{m}^{\prime}(s)\right\|_{2}^{2}+\left\|\nabla u_{m}(s)\right\|_{2}^{2}\right. \\
& \left.\quad+\left\|\nabla u_{m}^{\prime}(s)\right\|_{2}^{2}+\left\|\Delta u_{m}(s)\right\|_{2}^{2}\right) d s \\
& +C\left(T,\|\sigma\|_{L^{2}\left([0, T] \times \Omega ; H_{0}^{1}\right)},\|\sigma\|_{L^{2}\left([0, T] \times \Omega ; L^{\infty}\right)}\right) .
\end{aligned}
$$

Set

$$
y(t)=\left\|u_{m}^{\prime}(t)\right\|_{2}^{2}+\left\|\nabla u_{m}(t)\right\|_{2}^{2}+\left\|\nabla u_{m}^{\prime}(t)\right\|_{2}^{2}+\left\|\Delta u_{m}(t)\right\|_{2}^{2},
$$

and we can rewrite (39) as follows:

$$
\begin{aligned}
E \sup _{t \in[0, T]} y(t) \leq & C \int_{0}^{T} E \sup _{t \in[0, s]} y(t) d s \\
& -4 E \int_{0}^{T}\left(g_{\lambda}\left(u_{m}^{\prime}\right), u_{m}^{\prime}\right) d s+C,
\end{aligned}
$$

and due to Gronwall's inequality and (29), it is clear that

$$
\begin{gathered}
E\left(\sup _{t \in[0, T]}\left(\left\|u_{m}^{\prime}\right\|_{2}^{2}+\left\|\nabla u_{m}\right\|_{2}^{2}+\left\|\nabla u_{m}^{\prime}\right\|_{2}^{2}+\left\|\Delta u_{m}\right\|_{2}^{2}\right)\right. \\
\left.+\int_{0}^{T}\left(g_{\lambda}\left(u_{m}^{\prime}\right), u_{m}^{\prime}\right) d s\right) \leq C .
\end{gathered}
$$

Let $P_{m}$ be the orthogonal projection of $L^{2}(D)$ into the space Span $\left\{e_{1}, \ldots, e_{m}\right\}$, such that

$$
P_{m} \psi=\sum_{i=1}^{m}\left(\psi, e_{i}\right) e_{i}
$$

Define $M(t):=\int_{0}^{t} \sigma d W_{s}$, where $\sigma(x, t)$ is an $H_{0}^{1}(D) \cap L^{\infty}(D)$ valued progressively measurable such that (19) holds and $\{W(t, x): t \geq 0\}$ is an $H_{0}^{1}$-valued process; there is a subset $\Omega_{1} \in \Omega$ with $P\left(\Omega_{1}\right)=1$ such that for each $\omega \subseteq \Omega_{1}, M \in$ $C\left([0, T] ; H_{0}^{1}\right)$, and we have

$$
\begin{aligned}
\partial_{t}\left(u_{m}^{\prime}-\varepsilon P_{m} M(t)\right)= & -\int_{0}^{t} h(t-\tau) \Delta u_{m}(\tau) d \tau \\
& +\Delta u_{m}-P_{m} g_{\lambda}\left(u_{m}^{\prime}\right)+P_{m} f_{N}\left(u_{m}\right)
\end{aligned}
$$

for all $m \geq 1$. 
From (42), there is a subsequence $\left\{u_{m_{k}}\right\}_{k=1}^{\infty}$, for each $\omega \in$ $\Omega_{1}$, such that,

$$
\begin{array}{r}
u_{m_{k}} \rightarrow u, \quad \text { weakly star in } L^{\infty}\left(0, T ; H^{2} \cap H_{0}^{1}\right), \\
u_{m_{k}} \longrightarrow u, \quad \text { strongly in } C\left(0, T ; H_{0}^{1}\right), \\
u_{m_{k}}^{\prime} \rightarrow u^{\prime}, \quad \text { weakly star in } L^{\infty}\left(0, T ; H_{0}^{1}\right), \\
\text { for } u=u(\omega),
\end{array}
$$

and by the properties of relaxation function $h$ and Hölder's inequality, we get

$$
\begin{aligned}
& \left\|\int_{0}^{t} h(t-\tau) \Delta u_{m}(\tau) d \tau\right\|_{L^{q /(q-1)}\left(0, T ; H^{-1}\right)}^{q /(q-1)} \\
& \leq C \int_{0}^{T}\left(\int_{0}^{s} h(\tau) d \tau\right)^{1 /(q-1)} \\
& \quad \times \int_{0}^{s} h(s-\tau)\left\|\Delta u_{m}(\tau)\right\|_{H^{-1}}^{q /(q-1)} d \tau d s \\
& \leq C \sup _{t \in[0, T]}\left\|\Delta u_{m}(t)\right\|_{2}^{q /(q-1)} \int_{0}^{T}\left(\int_{0}^{s} h(\tau) d \tau\right)^{q /(q-1)} d s \leq C .
\end{aligned}
$$

From (15) and embedding theorem we have

$$
\left\|g_{\lambda}\left(u_{m_{k}}^{\prime}(t)\right)\right\|_{L^{q /(q-1)}\left(0, T ; H^{-1}\right)}^{q /(q-1)} \leq C .
$$

Together with (42)-(47), we obtain

$$
\left\|u_{m_{k}}^{\prime}(t)-\varepsilon P_{m_{k}} M(t)\right\|_{W^{1, q /(q-1)}\left(0, T ; H^{-1}\right)} \leq C,
$$

for all $k \geq 1$. By $(48)$ and $u_{m_{k}}^{\prime}(t) \rightarrow u^{\prime}(t)$ weakly star in $L^{\infty}(0$, $\left.T ; H_{0}^{1}\right)$, we have

$$
\begin{array}{r}
u_{m_{k}}^{\prime}(t)-\varepsilon P_{m_{k}} M(t) \longrightarrow u^{\prime}-\varepsilon M(t), \\
\text { strongly in } C\left([0, T] ; L^{2}\right) .
\end{array}
$$

This implies that there exists a subsequence still denoted by $\left\{u_{m_{k}}^{\prime}(t)\right\}$ such that

$$
u_{m_{k}}^{\prime}(t) \longrightarrow u^{\prime}(t), \quad \text { for almost all }(t, x) \in(0, T) \times D
$$

Due to (47) and Lemma 4, it is clear that

$$
g_{\lambda}\left(u_{m_{k}}^{\prime}(t)\right) \longrightarrow g_{\lambda}\left(u^{\prime}(t)\right),
$$$$
\text { weakly in } L^{q /(q-1)}((0, T) \times D) \text {. }
$$

Thus, $u=u(\omega)$ satisfies (17) in the sense of distributions over $(0, T) \times D$.
Next, we will prove the uniqueness of the solution. If there is another solution $\widetilde{u}(\omega)$ of $(17), \omega \in \Omega_{1}$, in the above sense, then $w=u-\tilde{u}$ satisfies

$$
\begin{gathered}
w_{t t}-\Delta w+\int_{0}^{t} h(t-\tau) \Delta w(\tau) d \tau+g_{\lambda}\left(u_{t}\right)-g_{\lambda}\left(\widetilde{u_{t}}\right) \\
=f_{N}(u)-f_{N}(\tilde{u}), \quad w(0)=0, w_{t}(0)=0 .
\end{gathered}
$$

Taking the inner product of (51) with $w^{\prime}(t)$ in $L^{2}(D)$, we obtain

$$
\begin{aligned}
\frac{1}{2} \frac{d}{d t}( & \left.\left\|w^{\prime}(t)\right\|_{2}^{2}+\|\nabla w(t)\|_{2}^{2}\right) \\
& +\int_{0}^{t} h(t-\tau)\left(\Delta w(\tau), w^{\prime}(t)\right) d \tau \\
& +\left(g_{\lambda}\left(u_{t}\right)-g_{\lambda}\left(\widetilde{u}_{t}\right), w^{\prime}(t)\right) \\
= & \left(f_{N}(u)-f_{N}(\tilde{u}), w^{\prime}(t)\right) .
\end{aligned}
$$

From (15), we have

$$
\left(g_{\lambda}\left(u_{t}\right)-g_{\lambda}\left(\widetilde{u_{t}}\right), w^{\prime}(t)\right) \geq 0 .
$$

By Lemma 1 and Hölder's inequality,

$$
\begin{aligned}
\left|\left(f_{N}(u)-f_{N}(\widetilde{u}), w^{\prime}(t)\right)\right| & \leq\left\|f_{N}(u)-f_{N}(\widetilde{u})\right\|_{2}\left\|w^{\prime}(t)\right\|_{2} \\
& \leq C_{N}\|\nabla w\|_{2}\left\|w^{\prime}(t)\right\|_{2} .
\end{aligned}
$$

Due to (30), we have

$$
\begin{aligned}
\int_{0}^{t} h(t-\tau)\left(\Delta w(\tau), w^{\prime}(t)\right) d \tau \\
=-\frac{d}{d t} \int_{0}^{t} h(t-\tau)(\nabla w(\tau), \nabla w(t)) d \tau \\
\quad+h(0)\|\nabla w(t)\|_{2}^{2}+\int_{0}^{t} h^{\prime}(t-\tau)(\nabla w(\tau), \nabla w(t)) d \tau
\end{aligned}
$$

Combining (52) with (55), similar to (32) and (34), we get

$$
\begin{aligned}
\left\|w^{\prime}\right\|_{2}^{2}+\|\nabla w\|_{2}^{2} \leq & C_{N} \int_{0}^{t}\left(\|\nabla w\|_{2}^{2}+\left\|w^{\prime}\right\|_{2}^{2}\right) d s \\
& +\int_{0}^{t} h(t-\tau)(\nabla w(\tau), \nabla w(t)) d \tau \\
& +\int_{0}^{t} \int_{0}^{s} h^{\prime}(s-\tau)(\nabla w(\tau), \nabla w(s)) d \tau d s \\
\leq & C_{N} \int_{0}^{t}\left(\|\nabla w\|_{2}^{2}+\left\|w^{\prime}\right\|_{2}^{2}\right) d s \\
& +\varepsilon\|\nabla w\|_{2}^{2}+C_{\varepsilon} \int_{0}^{t}\|\nabla w\|_{2}^{2} d s
\end{aligned}
$$


which implies $w=0$, that is, $u(\omega)=\tilde{u}(\omega)$. So $u=u(\omega)$ is well defined, for each $\omega \in \Omega_{1}$.

Finally, we state that $\left(u, u_{t}\right)$ is $\left(H^{2}(D) \cap H_{0}^{1}(D)\right) \times H_{0}^{1}(D)$ valued progressively measurable for any $0 \leq t \leq T$, and the energy inequality holds true; this can be established by the similar argument in $[28,31]$.

Moreover, we still fix $N>0$ and consider the following problem:

$$
\begin{gathered}
u_{t t}-\Delta u+\int_{0}^{t} h(t-\tau) \Delta u(\tau) d \tau+g\left(u_{t}\right) \\
=f_{N}(u)+\varepsilon \sigma(x, t) \partial_{t} W(t, x), \\
u(x, t)=0, \quad(x, t) \in \partial D \times(0, T), \\
u(x, 0)=u_{0}(x), \quad u_{t}(x, 0)=u_{1}(x), \quad x \in \bar{D} .
\end{gathered}
$$

The following lemma is important to prove the local existence of solution of (1).

Lemma 6 (see [28, 31]). Assume that (18), (19), and the conditions of h hold. Then there is a pathwise unique solution $u$ of (58) such that

$$
\begin{gathered}
u \in L^{2}\left(\Omega ; L^{\infty}\left(0, T ; H^{2} \cap H_{0}^{1}\right)\right) \cap L^{2}\left(\Omega ; C\left([0, T] ; H_{0}^{1}\right)\right) \\
u_{t} \in L^{2}\left(\Omega ; L^{\infty}\left(0, T ; H_{0}^{1}\right)\right) \cap L^{2}\left(\Omega ; C\left([0, T] ; L^{2}\right)\right) \\
u_{t} \in L^{q}((0, T) \times D) .
\end{gathered}
$$

From Lemmas 5 and 6, we state a local existence theorem of (1); the proof is standard; for more information we refer the readers to $[28,31]$.

Theorem 7 (see $[28,31])$. Assume that $\left(u_{0}, u_{t}\right) \in H_{0}^{1}(D) \times$ $L^{2}(D), E \int_{0}^{T}\|\sigma(t)\|_{2}^{2} d t<\infty,(6)$, and the conditions of $h$ hold; there is a pathwise unique local solution $u$ of (1) according to Definition 2 such that the following energy equation holds:

$$
\begin{aligned}
\left\|u^{\prime}(t)\right\|_{2}^{2}+\|\nabla u(t)\|_{2}^{2} \\
=\left\|u^{\prime}(0)\right\|_{2}^{2}+\|\nabla u(0)\|_{2}^{2} \\
\quad-2 \int_{0}^{t} \int_{D} \int_{0}^{s} h(t-\tau) \Delta u(\tau) d \tau u^{\prime}(s) d x d s \\
\quad+2 \int_{0}^{t} \int_{D}|u|^{p-2} u u^{\prime}(s) d x d s+2 \int_{0}^{t}\left(u^{\prime}(s), \varepsilon \sigma d W_{s}\right) \\
\quad-2 \int_{0}^{t} \int_{D}\left|u_{s}\right|^{q} d x d s+\varepsilon^{2} \sum_{j=1}^{\infty} \int_{0}^{t} \int_{D} \lambda_{j} e_{j}^{2} \sigma^{2} d x d s .
\end{aligned}
$$

\section{Blow Up}

In this section we prove our main result for $p>q$. For this purpose, we give refined restrictions on $\sigma(x, t)$ and relaxation function $h$ such that

$$
E \int_{0}^{\infty} \int_{D} \sigma^{2}(x, t) d x d t<\infty, \quad \int_{0}^{\infty} h(s) d s<\frac{p(p-2)}{(p-1)^{2}}
$$

Define an energy function

$$
\begin{aligned}
F(t)= & \frac{1}{2}\left\|u_{t}(t)\right\|_{2}^{2}+\frac{1}{2}\left(1-\int_{0}^{t} h(s) d s\right)\|\nabla u(t)\|_{2}^{2} \\
& +\frac{1}{2}(h \circ \nabla u)(t)-\frac{1}{p}\|u(t)\|_{p}^{p},
\end{aligned}
$$

where

$$
(h \circ \nabla u)(t)=\int_{0}^{t} h(t-s)\|u(t)-u(s)\|_{2}^{2} d s .
$$

For each $N$, introduce the stopping time $\tau_{N}$ by $\tau_{N}=$ $\inf \left\{t>0 ;\|\nabla u\|_{2} \geq N\right\}$, where $\tau_{N}$ is increasing in $N$, let $\tau_{\infty}=$ $\lim _{N \rightarrow \infty} \tau_{N}$.

In order to prove our blow-up result, we rewrite (1) as an equivalent Itô system

$$
\begin{gathered}
d u=v d t \\
d v=\left(\Delta u-\int_{0}^{t} h(t-\tau) \Delta u(\tau) d \tau-|v|^{q-2} v+|u|^{p-2} u\right) d t \\
+\varepsilon \sigma(x, t) d W(t, x), \\
u(x, t)=0, \quad(x, t) \in \partial D \times(0, T), \\
u(x, 0)=u_{0}(x), \quad v(x, 0)=v_{0}(x)=u_{1}(x), \quad x \in \bar{D},
\end{gathered}
$$

where $\left(u_{0}, u_{1}\right) \in H_{0}^{1} \times L^{2}$. Then the energy function $F(t)$ becomes

$$
\begin{aligned}
F(t)= & \frac{1}{2}\|v(t)\|_{2}^{2}+\frac{1}{2}\left(1-\int_{0}^{t} h(s) d s\right)\|\nabla u(t)\|_{2}^{2} \\
& +\frac{1}{2}(h \circ \nabla u)(t)-\frac{1}{p}\|u(t)\|_{p}^{p} .
\end{aligned}
$$

First we give a lemma. 
Lemma 8. Assume that (6), (61), and the conditions of h hold. Let $(u, v)$ be a solution of system (64) with initial data $\left(u_{0}, v_{0}\right) \in$ $H_{0}^{1} \times L^{2}$. Then we have

$$
\frac{d}{d t} E[F(t)] \leq-E\|v\|_{q}^{q}+\frac{1}{2} \varepsilon^{2} \sum_{j=1}^{\infty} E \int_{D} \lambda_{j} e_{j}^{2}(x) \sigma^{2}(x, t) d x
$$

$$
\begin{aligned}
& E[(u, v)(t)] \\
& =\left(u_{0}, v_{0}\right)-\int_{0}^{t} E\|\nabla u\|_{2}^{2} d s-\int_{0}^{t} E\left(u,|v|^{q-2} v\right) d s \\
& \quad+\int_{0}^{t} E\|u\|_{p}^{p} d s+E \int_{0}^{t} \int_{0}^{s} h(s-\tau)(\nabla u(\tau), \nabla u(s)) d \tau d s \\
& \quad+\int_{0}^{t} E\|v\|_{2}^{2} d s .
\end{aligned}
$$

Proof. Using Itô formula to $\|v\|_{2}^{2}$ and $(u, v)$, respectively, and taking the expectations, in the same way as our discussions in existence of solution to deal with the memory term, it is easy to get (66) and (67) (see [28]).

Let

$$
G(t)=\frac{\varepsilon^{2}}{2} \sum_{j=1}^{\infty} E \int_{0}^{t} \int_{D} \lambda_{j} e_{j}^{2}(x) \sigma^{2}(x, s) d x d s .
$$

Due to (61), we have

$$
\begin{aligned}
G(\infty) & =\frac{\varepsilon^{2}}{2} \sum_{j=1}^{\infty} E \int_{0}^{\infty} \int_{D} \lambda_{j} e_{j}^{2}(x) \sigma^{2}(x, s) d x d s \\
& \leq \frac{\varepsilon^{2}}{2} \operatorname{Tr}(R) C_{0}^{2} E \int_{0}^{\infty} \int_{D} \sigma^{2}(x, s) d x d s=E_{1}<\infty .
\end{aligned}
$$

We set $H(t):=G(t)-E[F(t)]$. Then, (66) implies that

$$
H^{\prime}(t)=G^{\prime}(t)-\frac{d}{d t} E[F(t)] \geq E\|v(t)\|_{q}^{q} \geq 0 .
$$

Lemma 9. Let $(u, v)$ be a solution of system (64). Then there exists a positive constant $C>0$ such that

$$
\begin{array}{r}
E\|u(t)\|_{p}^{s} \leq C\left[G(t)-H(t)-E\|v(t)\|_{2}^{2}\right. \\
\left.+E\|u(t)\|_{p}^{p}-\frac{1}{2}(h \circ \nabla u)(t)\right], \\
2 \leq s \leq p .
\end{array}
$$

Proof. If $\|u\|_{p} \leq 1$ then $\|u\|_{p}^{s} \leq\|u\|_{p}^{2} \leq C\|\nabla u\|_{2}^{2}$ by Sobolev embedding theorem. If $\|u\|_{p} \geq 1$ then $\|u\|_{p}^{s} \leq\|u\|_{p}^{p}$. Therefore, combination with the definition of energy function, we can get (71).
Theorem 10. Assume that (6), (61), and the conditions of $h$ hold. Let $(u, v)$ be a solution of system (64) with initial data $\left(u_{0}, v_{0}\right) \in H_{0}^{1} \times L^{2}$ satisfying

$$
F(0) \leq-(1+\beta) E_{1},
$$

where $\beta>0$ is an arbitrary constant. If $p>q$, then the solution $(u, v)$ and the lifespan $\tau_{\infty}$ defined above, either

(1) $P\left(\tau_{\infty}<+\infty\right)>0$, that is, $\|\nabla u(t)\|_{2}$ blows up in finite time with positive probability, or

(2) there exists a positive time $T^{*} \in\left(0, T_{0}\right]$ such that

$$
\lim _{t \rightarrow T^{*}} E[F(t)]=+\infty,
$$

where

$$
T_{0}=\frac{1-\alpha}{\alpha K L^{-\alpha /(1-\alpha)}(0)}
$$

$$
L(0)=H^{1-\alpha}(0)+\delta E\left(u_{0}, u_{1}\right)>0,
$$

and $\alpha, K$ are given in later.

Proof. For the lifespan $\tau_{\infty}$ of the solution $\{u(t) ; t \geq 0\}$ of (1) with $H_{0}^{1}$ norm, firstly we treat the case when $P\left(\tau_{\infty}=+\infty\right)<$ 1. Then, for sufficiently large $T>0$, by (70) and (72), we have

$$
\begin{aligned}
0<(1+\beta) E_{1} \leq-F(0)= & H(0) \leq H(t) \leq G(t) \\
& +\frac{1}{p}\|u\|_{p}^{p} \leq E_{1}+\frac{1}{p}\|u\|_{p}^{p} .
\end{aligned}
$$

Define $L(t)=H^{1-\alpha}(t)+\delta E(u, v)$, where

$$
0<\alpha<\min \left\{\frac{1}{2}, \frac{p-2}{2 p}, \frac{p-q}{p q}\right\}
$$

and $\delta$ is a very small constant determined in later.

Using (67) and (70), we obtain

$$
\begin{aligned}
& L^{\prime}(t)=(1-\alpha) H^{-\alpha}(t) H^{\prime}(t) \\
& +\delta\left[-E\|\nabla u\|_{2}^{2}-E\left(u,|v|^{q-2} v\right)+E\|u\|_{p}^{p}\right. \\
& \\
& \left.\quad+E \int_{0}^{t} h(t-\tau)(\nabla u(\tau), \nabla u(t)) d \tau+E\|v\|_{2}^{2}\right]
\end{aligned}
$$




$$
\begin{aligned}
& \geq(1-\alpha) H^{-\alpha}(t) E\|v\|_{q}^{q}+\delta p[H(t)-G(t)+E F(t)] \\
& -\delta E\|\nabla u\|_{2}^{2}+\delta E\|v\|_{2}^{2}-\delta E\left(u,|v|^{q-2} v\right)+\delta E\|u\|_{p}^{p} \\
& \quad+\delta \int_{0}^{t} h(t-\tau)(\nabla u(\tau), \nabla u(t)) d \tau \\
& \geq(1-\alpha) H^{-\alpha}(t) E\|v\|_{q}^{q} \\
& \quad+\delta p H(t)+\delta\left(\frac{p}{2}-1\right) E\|\nabla u\|_{2}^{2} \\
& \quad+\delta\left(\frac{p}{2}+1\right) E\|v\|_{2}^{2}-\delta E\left(u,|v|^{q-2} v\right) \\
& \quad+\delta E \int_{0}^{t} h(t-\tau)(\nabla u(\tau), \nabla u(t)) d \tau \\
& \quad+\frac{\delta p}{2}(h \circ \nabla u)(t) \\
& \quad-\frac{\delta p}{2} E \int_{0}^{t} h(\tau) d \tau\|\nabla u(t)\|_{2}^{2}-\delta p G(t), \\
& \quad \delta E \int_{0}^{t} h(t-\tau)(\nabla u(\tau), \nabla u(t)) d \tau \\
& =\delta E \int_{0}^{t} h(t-\tau)(\nabla u(\tau)-\nabla u(t), \nabla u(t)) d \tau \\
& \quad+\delta E \int_{0}^{t} h(\tau) d \tau\|\nabla u(t)\|_{2}^{2},
\end{aligned}
$$

and by the Hölder's inequality,

$$
\begin{gathered}
\delta E \int_{0}^{t} h(t-\tau)(\nabla u(\tau)-\nabla u(t), \nabla u(t)) d \tau \\
\geq-\delta E\left[\frac{p}{2} \int_{0}^{t} h(t-\tau)\|\nabla u(\tau)-\nabla u(t)\|_{2}^{2} d \tau\right. \\
\left.\quad+\frac{1}{2 p} \int_{0}^{t} h(\tau) d \tau\|\nabla u(t)\|_{2}^{2}\right] \\
=-E \frac{\delta p}{2}(h \circ \nabla u)(t)-\frac{\delta}{2 p} E \int_{0}^{t} h(\tau) d \tau\|\nabla u(t)\|_{2}^{2} .
\end{gathered}
$$

Inserting (78) and (79) into (77), we get

$$
\begin{aligned}
L^{\prime}(t) \geq & (1-\alpha) H^{-\alpha}(t) E\|v\|_{q}^{q}+\delta p H(t) \\
& +\delta\left(\frac{p}{2}-1\right) E\|\nabla u\|_{2}^{2}+\delta\left(\frac{p}{2}+1\right) E\|v\|_{2}^{2} \\
& -\delta p G(t)+\delta\left(1-\frac{p^{2}+1}{2 p}\right) \\
& \times \int_{0}^{t} h(\tau) d \tau\|\nabla u(t)\|_{2}^{2}-\delta E\left(u,|v|^{q-2} v\right) .
\end{aligned}
$$

From $q<p$, by $E\|u\|_{q}^{q} \leq C E\|u\|_{p}^{q}$ and Hölder's inequality, we obtain the estimate of the last term in (80)

$$
\begin{aligned}
\left|E\left(u,|v|^{q-2} v\right)\right| \leq & \left(E\|v\|_{q}^{q}\right)^{(q-1) / q}\left(E\|u\|_{q}^{q}\right)^{1 / q} \\
\leq & C\left(E\|v\|_{q}^{q}\right)^{(q-1) / q}\left(E\|u\|_{p}^{q}\right)^{1 / q} \\
\leq & C\left(E\|v\|_{q}^{q}\right)^{(q-1) / q}\left(E\|u\|_{p}^{p}\right)^{1 / p} \\
\leq & C\left(E\|v\|_{q}^{q}\right)^{(q-1) / q}\left(E\|u\|_{p}^{p}\right)^{1 / q} \\
& \times\left(E\|u\|_{p}^{p}\right)^{(1 / p)-(1 / q)},
\end{aligned}
$$

and the Young's inequality implies that

$$
\left(E\|v\|_{q}^{q}\right)^{(q-1) / q}\left(E\|u\|_{p}^{p}\right)^{1 / q} \leq \frac{q-1}{q} \mu E\|v\|_{q}^{q}+\frac{\mu^{1-q}}{q} E\|u\|_{p}^{p},
$$

where $\rho=p \beta /(1+\beta)$. We assume $H(0)>1$, (83), and (76) imply that

$$
\begin{aligned}
\left(E\|u\|_{p}^{p}\right)^{(1 / p)-(1 / q)} & \leq \rho^{(1 / p)-(1 / q)} H^{(1 / p)-(1 / q)}(t) \\
& \leq \rho^{(1 / p)-(1 / q)} H^{-\alpha}(t) \leq \rho^{(1 / p)-(1 / q)} H^{-\alpha}(0) .
\end{aligned}
$$

Combining (82) with (84), we arrive that

$$
\begin{aligned}
\left|E\left(u,|v|^{q-2} v\right)\right| \leq & a_{1} \frac{q-1}{q} \mu E\|v\|_{q}^{q} H^{-\alpha}(t) \\
& +a_{1} \frac{\mu^{1-q}}{q} E\|u\|_{p}^{p} H^{-\alpha}(0),
\end{aligned}
$$

where $a_{1}=C \rho^{(1 / p)-(1 / q)}$.

Hence, substituting (85) into (80),

$$
\begin{aligned}
L^{\prime}(t) \geq & \left(1-\alpha-a_{1} \frac{q-1}{q} \mu \delta\right) H^{-\alpha}(t) E\|v\|_{q}^{q} \\
& +\delta p H(t)+\delta\left(\frac{p}{2}-1\right) E\|\nabla u\|_{2}^{2}+\delta\left(\frac{p}{2}+1\right) E\|v\|_{2}^{2} \\
& -\delta p G(t)+\delta\left(1-\frac{p^{2}+1}{2 p}\right) \\
& \times \int_{0}^{t} h(\tau) d \tau\|\nabla u(t)\|_{2}^{2}-\delta a_{1} \frac{\mu^{1-q}}{q} E\|u\|_{p}^{p} H^{-\alpha}(0) .
\end{aligned}
$$


By Lemma 9 with $s=p$ and (86), we have

$$
\begin{aligned}
& L^{\prime}(t) \geq\left(1-\alpha-a_{1} \frac{q-1}{q} \mu \delta\right) H^{-\alpha}(t) E\|v\|_{q}^{q} \\
& +\delta p H(t)+\delta\left(\frac{p}{2}-1\right) E\|\nabla u\|_{2}^{2} \\
& +\delta\left(\frac{p}{2}+1\right) E\|v\|_{2}^{2}-\delta p G(t) \\
& +\delta\left(1-\frac{p^{2}+1}{2 p}\right) \int_{0}^{t} h(\tau) d \tau\|\nabla u(t)\|_{2}^{2} \\
& -\delta a_{2} \mu^{1-q}\left[G(t)-H(t)-E\|v\|_{2}^{2}\right. \\
& \left.+E\|u\|_{p}^{p}-\frac{1}{2}(h \circ \nabla u)\right] \\
& \geq\left(1-\alpha-a_{1} \frac{q-1}{q} \mu \delta\right) H^{-\alpha}(t) E\|v\|_{q}^{q} \\
& +\delta\left(p+a_{2} \mu^{1-q}\right) H(t)-\delta\left(p+a_{2} \mu^{1-q}\right) G(t) \\
& +\delta\left(\frac{p}{2}+1+a_{2} \mu^{1-q}\right) E\|v\|_{2}^{2} \\
& -\delta a_{2} \mu^{1-q} E\|u\|_{p}^{p}+\frac{\delta a_{2} \mu^{1-q}}{2} E(h \circ \nabla u) \\
& +\delta\left[\frac{p}{2}-1+\left(1-\frac{p^{2}+1}{2 p}\right) \int_{0}^{t} h(\tau) d \tau\right]\|\nabla u(t)\|_{2}^{2},
\end{aligned}
$$

where $a_{2}=C a_{1}\left(H^{-\alpha}(0) / q\right)$.

Note that

$$
H(t) \geq G(t)+\frac{1}{p}\|u\|_{p}^{p}-\frac{1}{2}\|v\|_{2}^{2}-\frac{1}{2}\|\nabla u\|_{2}^{2}-\frac{1}{2}(h \circ \nabla u)(t),
$$

and denote

$$
a_{3}=\frac{p}{2}-1+\left(1-\frac{p^{2}+1}{2 p}\right) \int_{0}^{\infty} h(\tau) d \tau>0
$$

We write $p=2 a_{4}+\left(p-2 a_{4}\right)$, where $a_{4}=\min \left\{a_{1}, a_{3}\right\}$, the estimate (87) yields

$$
\begin{aligned}
L^{\prime}(t) \geq & \left(1-\alpha-a_{1} \frac{q-1}{q} \mu \delta\right) H^{-\alpha}(t) E\|v\|_{q}^{q} \\
& +\delta\left(p-2 a_{4}+a_{2} \mu^{1-q}\right) H(t) \\
& -\delta\left(p-2 a_{4}+a_{2} \mu^{1-q}\right) G(t) \\
& +\delta\left(\frac{p}{2}+1-a_{4}+a_{2} \mu^{1-q}\right) E\|v\|_{2}^{2}
\end{aligned}
$$

$$
\begin{aligned}
& +\delta\left(a_{2} \mu^{1-q}+\frac{2 a_{4}}{p}\right) E\|u\|_{p}^{p} \\
& +\delta\left(\frac{a_{2} \mu^{1-q}}{2}-a_{4}\right) E(h \circ \nabla u) \\
& +\delta\left(a_{3}-a_{4}\right) E\|\nabla u(t)\|_{2}^{2} .
\end{aligned}
$$

From (72) and (75), we obtain

$$
\begin{aligned}
\left(p-2 a_{4}+a_{2} \mu^{1-q}\right) G(t) & \leq\left(p-2 a_{4}+a_{2} \mu^{1-q}\right) E_{1} \\
& \leq \frac{p-2 a_{4}+a_{2} \mu^{1-q}}{1+\beta} H(t) .
\end{aligned}
$$

Substituting (91) into (90), we get

$$
\begin{aligned}
L^{\prime}(t) \geq & \left(1-\alpha-a_{1} \frac{q-1}{q} \mu \delta\right) H^{-\alpha}(t) E\|v\|_{q}^{q} \\
& +\delta\left(p-2 a_{4}+a_{2} \mu^{1-q}\right) \frac{\beta}{1+\beta} H(t) \\
& +\delta\left(\frac{p}{2}+1-a_{4}+a_{2} \mu^{1-q}\right) E\|v\|_{2}^{2} \\
& +\delta\left(a_{2} \mu^{1-q}+\frac{2 a_{4}}{p}\right) E\|u\|_{p}^{p} \\
& +\delta\left(\frac{a_{2} \mu^{1-q}}{2}-a_{4}\right) E(h \circ \nabla u) \\
& +\delta\left(a_{3}-a_{4}\right)\|\nabla u(t)\|_{2}^{2} .
\end{aligned}
$$

Next, we can choose $\mu$ large enough so that (92) becomes

$$
\begin{gathered}
L^{\prime}(t) \geq\left(1-\alpha-a_{1} \frac{q-1}{q} \mu \delta\right) H^{-\alpha}(t) E\|v\|_{q}^{q} \\
+\delta \gamma\left(H(t)+E\|v\|_{2}^{2}+E\|u\|_{p}^{p}\right. \\
\left.-E(h \circ \nabla u)+\|\nabla u(t)\|_{2}^{2}\right),
\end{gathered}
$$

where $\gamma>0$ is the minimum of the coefficients of $H(t), E\|v\|_{2}^{2}$, $E\|u\|_{p}^{p}, E(h \circ \nabla u)$, and $\|\nabla u(t)\|_{2}^{2}$ in (93). Once $\mu$ is fixed, we pick $\delta$ small enough so that

$$
1-\alpha-a_{1} \frac{q-1}{q} \mu \delta \geq 0 .
$$

Therefore, (93) takes the form

$$
\begin{aligned}
L^{\prime}(t) \geq \delta \gamma\left(H(t)+E\|v\|_{2}^{2}+E\|u\|_{p}^{p}\right. \\
\left.-E(h \circ \nabla u)+\|\nabla u(t)\|_{2}^{2}\right) \geq 0 .
\end{aligned}
$$

Consequently we have

$$
L(t) \geq L(0)=H^{1-\alpha}(0)+\delta E\left(u_{0}, u_{1}\right)>0, \quad \forall t \geq 0 .
$$


Since

$$
\left|E \int_{D} u v d x\right| \leq C\left(E\|u\|_{p}^{2}\right)^{1 / 2}\left(E\|v\|_{2}^{2}\right)^{1 / 2}
$$

it implies that

$$
\left|E \int_{D} u v d x\right|^{1 /(1-\alpha)} \leq C\left\{\left(E\|u\|_{p}^{2}\right)^{\eta / 2(1-\alpha)}+\left(E\|v\|_{2}^{2}\right)^{\zeta / 2(1-\alpha)}\right\}
$$

for $(1 / \eta)+(1 / \zeta)=1$

We choose $\zeta=2(1-\alpha), \eta=2(1-\alpha) /(1-2 \alpha)$; then $\eta / 2(1-$ $\alpha)=1 /(1-2 \alpha) \leq p / 2$, by (76) and (98) becomes

$$
\left|E \int_{D} u v d x\right|^{1 /(1-\alpha)} \leq C\left\{E\|u\|_{p}^{2 /(1-2 \alpha)}+E\|v\|_{2}^{2}\right\} .
$$

Using Lemma 9 with $s=2 /(1-2 \alpha)$, we obtain

$$
\begin{aligned}
& \left|E \int_{D} u v d x\right|^{1 /(1-\alpha)} \\
& \quad \leq C\left(H(t)+E\|\nabla u\|_{2}^{2}+E\|v\|_{2}^{2}+E\|u\|_{p}^{p}+E(h \circ \nabla u)\right)
\end{aligned}
$$

for all $t \geq 0$.

Therefore we have

$$
\begin{aligned}
L^{1 /(1-\alpha)}(t) & =\left(H^{1-\alpha}(t)+\delta E(u, v)\right)^{1 /(1-\alpha)} \\
\leq & 2^{1 /(1-\alpha)}\left(H(t)+\delta^{1 /(1-\alpha)}\left|E \int_{D} u v d x\right|^{1 /(1-\alpha)}\right) \\
\leq & C\left(H(t)+E\|\nabla u\|_{2}^{2}+E\|v\|_{2}^{2}\right. \\
& \left.+E\|u\|_{p}^{p}+E(h \circ \nabla u)\right)
\end{aligned}
$$

for all $t \geq 0$. Combining (95) and (101),

$$
L^{\prime}(t) \geq K L^{1 /(1-\alpha)}(t), \quad \forall t \geq 0,
$$

where $K$ is a positive constant depending only on $C$ and $\delta \gamma$; then it yields

$$
L^{\alpha /(1-\alpha)}(t) \geq \frac{1-\alpha}{(1-\alpha) L^{-\alpha /(1-\alpha)}(0)-\alpha K t} .
$$

Let

$$
T_{0}=\frac{1-\alpha}{\alpha K L^{-\alpha /(1-\alpha)}(0)} .
$$

Then $L(t) \rightarrow \infty$ as $t \rightarrow T_{0}$. This means that there exists a positive time $T^{*} \in\left(0, T_{0}\right]$ such that

$$
\lim _{t \rightarrow T^{*}} E[F(t)]=+\infty \text {. }
$$

As for the case when $P\left(\tau_{\infty}=+\infty\right)<1$ (i.e., $P\left(\tau_{\infty}<+\infty\right)>$ $0)$, then $\|\nabla u(t)\|_{2}$ blows up in finite time $T^{*} \in\left(0, \tau_{\infty}\right]$ with positive probability.

The proof of Theorem 10 is completed.
Remark 11. (1) In the deterministic case of $\varepsilon=0$, it is well known that for $\left(u_{0}, u_{1}\right) \in H_{0}^{1} \times L^{2}$, the condition $F(0)<0$ and $\int_{0}^{\infty} h(s) d s<p(p-2) /(p-1)^{2}$ already imply that the solution blows up in finite time (see, e.g., [11]). In the stochastic case of $\varepsilon>0$, to balance the influence of $W(t, x)$ such that the local solution of (1) blows up with positive probability or is explosive in $L^{2}$ sense, the initial energy and relaxation function should be satisfied that $F(0) \leq-(1+\beta) E_{1}$, and $\int_{0}^{\infty} h(s) d s<$ $p(p-2) /(p-1)^{2}$.

(2) Our results have included the case which is without viscoelastic term (i.e., $h=0$ satisfied (61)).

\section{Global Existence}

In this section we show that solution of (1) is global if $q \geq p$. We use the Borel-Cantelli Lemma to prove the existence of global solution. For this aim, we introduce an energy function

$$
e(u(t))=\left\|u_{t}(t)\right\|_{2}^{2}+\|\nabla u(t)\|_{2}^{2}+\|u(t)\|_{p}^{p}+(h \circ \nabla u)(t) .
$$

Theorem 12. Assume that $(1),\left(u_{0}, u_{1}\right) \in H_{0}^{1}(D) \times L^{2}(D)$, $E \int_{0}^{T}\|\sigma(t)\|_{2}^{2} d t<\infty$, and the conditions of h hold. If $q \geq p, u(t)$ is a solution of (1) with initial data $\left(u_{0}, u_{1}\right) \in H_{0}^{1} \times L^{2}$ according to Definition 2 on the interval $[0, T]$; then for any $T>0$,

$$
E \sup _{0 \leq t \leq T} e(u(t))<\infty
$$

Proof. For any $T>0$, we will show that $u_{N}(t)=u\left(t \wedge \tau_{N}\right) \rightarrow$ $u$ (a.s.) as $N \rightarrow \infty$ for any $t \leq T$, so that the local solution becomes a global solution where $\tau_{N}$ is a stopping time which is defined in Section 4. Similar to [28], by the Theorem 7, for $t \in\left[0, T \wedge \tau_{N}\right), u(t)=u_{N}(t)=u\left(t \wedge \tau_{N}\right)$ is the local solution of (1), so the following energy equation holds:

$$
\begin{aligned}
e\left(u\left(t \wedge \tau_{N}\right)\right)= & e\left(u_{0}\right)+(h \circ \nabla u)\left(t \wedge \tau_{N}\right) \\
& -2 \int_{0}^{t \wedge \tau_{N}} \int_{D} \int_{0}^{s} h(t-\tau) \Delta u(\tau) d \tau u^{\prime}(s) d x d s \\
& +4 \int_{0}^{t \wedge \tau_{N}} \int_{D}|u|^{p-2} u u^{\prime}(s) d x d s \\
& +2 \int_{0}^{t \wedge \tau_{N}}\left(u^{\prime}(s), \varepsilon \sigma d W_{s}\right) \\
& -2 \int_{0}^{t \wedge \tau_{N}} \int_{D}\left|u_{t}\right|^{q} d x d s \\
& +\varepsilon^{2} \sum_{j=1}^{\infty} \int_{0}^{t \wedge \tau_{N}} \int_{D} \lambda_{j} e_{j}^{2} \sigma^{2} d x d s .
\end{aligned}
$$


Using Hölder's inequality, Young's inequality, and embedding theorem, we have

$$
\begin{aligned}
\left.\left|\int_{D}\right| u\right|^{p-2} u u^{\prime}(t) d x \mid \leq & \|u\|_{p}^{p-1}\left\|u_{t}\right\|_{p} \leq \nu\left\|u_{t}\right\|_{p}^{p} \\
& +C_{\nu}\|u\|_{p}^{p} \leq C \nu\left\|u_{t}\right\|_{q}^{p}+C_{\nu}\|u\|_{p}^{p}
\end{aligned}
$$

where $v>0, C$ is the embedding constant, and $C_{\nu}$ is a constant depending on $v$, consequently; we have

$$
\begin{aligned}
& e\left(u\left(t \wedge \tau_{N}\right)\right) \\
& \leq e\left(u_{0}\right)+(h \circ \nabla u)\left(t \wedge \tau_{N}\right) \\
& \quad-2 \int_{0}^{t \wedge \tau_{N}} \int_{D} \int_{0}^{s} h(s-\tau) \Delta u(\tau) d \tau u^{\prime}(s) d x d s \\
& \quad+4 C v \int_{0}^{t \wedge \tau_{N}}\left\|u_{t}\right\|_{q}^{p} d s+4 C_{v} \int_{0}^{t \wedge \tau_{N}}\|u\|_{p}^{p} d s \\
& \quad+2 \int_{0}^{t \wedge \tau_{N}}\left(u^{\prime}(s), \varepsilon \sigma d W_{s}\right)-2 \int_{0}^{t \wedge \tau_{N}}\left\|u_{t}\right\|_{q}^{q} d s \\
& \quad+\varepsilon^{2} \operatorname{Tr}(R) C_{0}^{2} \int_{0}^{t \wedge \tau_{N}}\|\sigma\|_{2}^{2} d s .
\end{aligned}
$$

Since $q \geq p$, we distinguish two cases.

(1) Either $\left\|u_{t}\right\|_{q}^{q}>1$ so we choose $v$ so small that $-2\left\|u_{t}\right\|_{q}^{q}+$ $4 C \nu\left\|u_{t}\right\|_{q}^{p} \leq 0$.

(2) Or $\left\|u_{t}\right\|_{q}^{q} \leq 1$; in this case, we have $-2\left\|u_{t}\right\|_{q}^{q}+$ $4 C \nu\left\|u_{t}\right\|_{q}^{p} \leq 4 C \nu$.

Hence, in either case, we have

$$
\begin{aligned}
e\left(u\left(t \wedge \tau_{N}\right)\right) \leq & e\left(u_{0}\right)+(h \circ \nabla u)\left(t \wedge \tau_{N}\right) \\
& -2 \int_{0}^{t \wedge \tau_{N}} \int_{D} \int_{0}^{s} h(s-\tau) \Delta u(\tau) d \tau u_{t} d x d s \\
& +4 C \nu\left(t \wedge \tau_{N}\right)+2 \int_{0}^{t \wedge \tau_{N}}\left(u^{\prime}(s), \varepsilon \sigma d W_{s}\right) \\
& +4 C_{v} \int_{0}^{t \wedge \tau_{N}}\|u\|_{p}^{p} d s \\
& +\varepsilon^{2} \operatorname{Tr}(R) C_{0}^{2} \int_{0}^{t \wedge \tau_{N}}\|\sigma\|_{2}^{2} d s .
\end{aligned}
$$

Using the conditions of $h$, we obtain

$$
\begin{aligned}
& -\int_{D} \int_{0}^{s} h(s-\tau)(\Delta u(\tau)) d \tau u_{t} d x \\
& \quad=\int_{0}^{s} h(s-\tau) \int_{D} \nabla u(\tau) \nabla u_{t}(s) d x d \tau \\
& \quad=\int_{0}^{s} h(s-\tau) \int_{D}\left(\nabla u(\tau)-\nabla u_{t}(s)\right) \nabla u_{t}(s) d x d \tau
\end{aligned}
$$

$$
\begin{aligned}
& +\int_{0}^{s} h(s-\tau) \int_{D} \nabla u_{t}(s) \nabla u_{t}(s) d x d \tau \\
= & -\frac{1}{2} \int_{0}^{s} h(s-\tau) \frac{d}{d s} \int_{D}\left|\nabla u(\tau)-\nabla u_{t}(s)\right|^{2} d x d \tau \\
& +\frac{1}{2} \int_{0}^{s} h(\tau) \frac{d}{d s} \int_{D}|\nabla u(\tau)|^{2} d x d \tau \\
= & \frac{1}{2} \frac{d}{d s}\left(\int_{0}^{s} h(\tau) d \tau\|\nabla u(s)\|_{2}^{2}-(h \circ \nabla u)(s)\right) \\
& +\frac{1}{2}\left(h^{\prime}(s) \circ \nabla u\right)(s)-\frac{1}{2} h(s)\|\nabla u(s)\|_{2}^{2} \\
\leq & \frac{1}{2} \frac{d}{d s}\left(\int_{0}^{s} h(\tau) d \tau\|\nabla u(s)\|_{2}^{2}-(h \circ \nabla u)(s)\right)
\end{aligned}
$$

which implies that

$$
\begin{gathered}
-2 \int_{0}^{t \wedge \tau_{N}} \int_{D} \int_{0}^{s} h(s-\tau)(\Delta u(\tau)) d \tau u_{t}(s) d x d s \\
\leq \int_{0}^{t \wedge \tau_{N}} h(\tau) d \tau\|\nabla u(t)\|_{2}^{2}-(h \circ \nabla u)\left(t \wedge \tau_{N}\right) .
\end{gathered}
$$

Consequently we have

$$
\begin{aligned}
e\left(u\left(t \wedge \tau_{N}\right)\right) \leq & e\left(u_{0}\right)+\int_{0}^{t \wedge \tau_{N}} h(\tau) d \tau\|\nabla u(t)\|_{2}^{2} \\
& +4 C \nu\left(t \wedge \tau_{N}\right)+2 \int_{0}^{t \wedge \tau_{N}}\left(u^{\prime}(s), \varepsilon \sigma d W_{s}\right) \\
& +4 C_{\nu} \int_{0}^{t \wedge \tau_{N}}\|u\|_{p}^{p} d s \\
& +\varepsilon^{2} \operatorname{Tr}(R) C_{0}^{2} \int_{0}^{t \wedge \tau_{N}}\|\sigma\|_{2}^{2} d s .
\end{aligned}
$$

Taking the expectation of (114), we get

$$
\begin{aligned}
E e\left(u\left(t \wedge \tau_{N}\right)\right) \leq & e\left(u_{0}\right)+4 C \nu\left(t \wedge \tau_{N}\right) \\
& +C \int_{0}^{t \wedge \tau_{N}} E e(u(s)) d s \\
& +\varepsilon^{2} \operatorname{Tr}(R) C_{0}^{2} \int_{0}^{t \wedge \tau_{N}} E\|\sigma\|_{2}^{2} d s .
\end{aligned}
$$

The Gronwall's inequality implies that

$$
E e\left(u\left(t \wedge \tau_{N}\right)\right) \leq\left(e\left(u_{0}\right)+C T\right) e^{C T} \leq C_{T} .
$$

On the other hand, we have

$$
\begin{aligned}
E e\left(u\left(t \wedge \tau_{N}\right)\right) & \geq E\left(I_{\tau_{N}<T} e\left(u\left(\tau_{N}\right)\right)\right) \\
& \geq C E\left(I_{\tau_{N}<T}\left\|\nabla u\left(\tau_{N}\right)\right\|_{2}^{2}\right) \geq C^{2} P\left(\tau_{N}<T\right),
\end{aligned}
$$


where $I(\cdot)$ denotes the indicator function. In view of (116) and (117), we get

$$
P\left(\tau_{N}<T\right) \leq \frac{C_{T}}{N^{2}}
$$

The Borel-Cantelli lemma implies that $P\left(\tau_{\infty}<T\right)=0$ for any $T>0$. This shows that $u=\lim _{N \rightarrow \infty} u_{N}(t)$ is the global solution.

\section{Conflict of Interests}

The authors declare that there is no conflict of interests regarding the publication of this paper.

\section{Acknowledgments}

This work is supported by NSF of China 11272277 and 11226188 and FRF for the Central Universities of China $2013 Z Z G H 027$.

\section{References}

[1] A. Haraux and E. Zuazua, "Decay estimates for some semilinear damped hyperbolic problems," Archive for Rational Mechanics and Analysis, vol. 150, no. 2, pp. 191-206, 1988.

[2] M. Kopáčková, "Remarks on bounded solutions of a semilinear dissipative hyperbolic equation," Commentationes Mathematicae Universitatis Carolinae, vol. 30, no. 4, pp. 713-719, 1989.

[3] J. M. Ball, "Remarks on blow-up and nonexistence theorems for nonlinear evolution equations," The Quarterly Journal of Mathematics, vol. 28, no. 112, pp. 473-486, 1977.

[4] V. K. Kalantarov and O. A. Ladyzhenskaya, "The occurrence of collapse for quasilinear equations of parabolic and hyperbolic types," Journal of Soviet Mathematics, vol. 10, no. 1, pp. 53-70, 1978.

[5] H. A. Levine, "Some additional remarks on the nonexistence of global solutions to nonlinear wave equations," SIAM Journal on Mathematical Analysis, vol. 5, pp. 138-146, 1974.

[6] H. A. Levine, S. R. Park, and J. Serrin, "Global existence and global nonexistence of solutions of the Cauchy problem for a nonlinearly damped wave equation," Journal of Mathematical Analysis and Applications, vol. 228, no. 1, pp. 181-205, 1998.

[7] V. Georgiev and G. Todorova, "Existence of a solution of the wave equation with nonlinear damping and source terms," Journal of Differential Equations, vol. 109, no. 2, pp. 295-308, 1994.

[8] C. O. Alves, M. M. Cavalcanti, V. N. D. Cavalcanti, M. A. Rammaha, and D. Toundykov, "On existence, uniform decay rates and blow up for solutions of systems of nonlinear wave equations with damping and source terms," Discrete and Continuous Dynamical Systems. Series S, vol. 2, no. 3, pp. 583-608, 2009.

[9] M. A. Rammaha, "The influence of damping and source terms on solutions of nonlinear wave equations," Boletim da Sociedade Paranaense de Matemática, vol. 25, no. 1-2, pp. 77-90, 2007.

[10] M. Kafini and S. A. Messaoudi, "A blow-up result in a Cauchy viscoelastic problem," Applied Mathematics Letters, vol. 21, no. 6, pp. 549-553, 2008.

[11] S. A. Messaoudi, "Blow up and global existence in a nonlinear viscoelastic wave equation," Mathematische Nachrichten, vol. 260, pp. 58-66, 2003.
[12] S. A. Messaoudi, "Blow-up of positive-initial-energy solutions of a nonlinear viscoelastic hyperbolic equation," Journal of Mathematical Analysis and Applications, vol. 320, no. 2, pp. 902915, 2006.

[13] H. Song and C. Zhong, "Blow-up of solutions of a nonlinear viscoelastic wave equation," Nonlinear Analysis: Real World Applications, vol. 11, no. 5, pp. 3877-3883, 2010.

[14] R. Ikehata, "Some remarks on the wave equations with nonlinear damping and source terms," Nonlinear Analysis: Theory, Methods \& Applications A, vol. 27, no. 10, pp. 1165-1175, 1996.

[15] M. Aassila, M. M. Cavalcanti, and V. N. D. Cavalcanti, "Existence and uniform decay of the wave equation with nonlinear boundary damping and boundary memory source term," Calculus of Variations and Partial Differential Equations, vol. 15, no. 2, pp. 155-180, 2002.

[16] M. M. Cavalcanti, V. N. D. Cavalcanti, J. S. P. Filho, and J. A. Soriano, "Existence and uniform decay rates for viscoelastic problems with nonlinear boundary damping," Differential and Integral Equations, vol. 14, no. 1, pp. 85-116, 2001.

[17] M. M. Cavalcanti, V. N. D. Cavalcanti, and P. Martinez, "Existence and decay rate estimates for the wave equation with nonlinear boundary damping and source term," Journal of Differential Equations, vol. 203, no. 1, pp. 119-158, 2004.

[18] M. M. Cavalcanti, V. N. D. Cavalcanti, and F. A. F. Nascimento, "Asymptotic stability of the wave equation on compact manifolds and locally distributed viscoelastic dissipation," Proceedings of the American Mathematical Society, vol. 141, no. 9, pp. 3183-3193, 2013.

[19] P.-L. Chow, Stochastic Partial Differential Equations, Chapman \& Hall/CRC, NewYork, 2007.

[20] S. L. Cheng, Y. T. Guo, and Y. B. Tang, "Stochastic nonlinear thermoelastic system coupled sine-Gordon equation driven by jump noise," Abstract and Applied Analysis, vol. 2014, Article ID 403528, 12 pages, 2014.

[21] G. Da Prato and J. Zabczyk, Stochastic Equations in Infinite Dimensions, Cambridge University Press, Cambridge, UK, 1996.

[22] G. Wang and Y. B. Tang, "Fractal dimension of a random invariant set and applications," Journal of Applied Mathematics, vol. 2013, Article ID 415764, 5 pages, 2013.

[23] G. Wang and Y. B. Tang, " $\left(L^{2}, H^{1}\right)$-random attractors for stochastic reaction-diffusion equation on unbounded domains," Abstract and Applied Analysis, vol. 2013, Article ID 279509, 23 pages, 2013.

[24] G. Wang and Y. B. Tang, "Random attractors for stochastic reaction-diffusion equations with multiplicative noise in $H_{0}^{1}$," Mathematische Nachrichten, 2014.

[25] M. Wang and Y. B. Tang, "Attractors in $H^{2}$ and $L^{2 p-2}$ for reaction diffusion equations on unbounded domains," Communications on Pure and Applied Analysis, vol. 12, no. 2, pp. 1111-1121, 2013.

[26] E. Wu and Y. B. Tang, "Random perturbations of reaction-diffusion waves in biology," Wave Motion, vol. 49, no. 7, pp. 632637, 2012.

[27] L. Bo, D. Tang, and Y. Wang, "Explosive solutions of stochastic wave equations with damping on $R^{d}$," Journal of Differential Equations, vol. 244, no. 1, pp. 170-187, 2008.

[28] H. Gao, F. Liang, and B. Guo, "Stochastic wave equations with nonlinear damping and source terms," Infinite Dimensional Analysis, Quantum Probability and Related Topics, vol. 16, no. 2, Article ID 1350013, 29 pages, 2013. 
[29] T. Wei and Y. Jiang, "Stochastic wave equations with memory," Chinese Annals of Mathematics B, vol. 31, no. 3, pp. 329-342, 2010.

[30] F. Liang and H. Gao, "Explosive solutions of stochastic viscoelastic wave equations with damping," Reviews in Mathematical Physics, vol. 23, no. 8, pp. 883-902, 2011.

[31] J. U. Kim, "On the stochastic wave equation with nonlinear damping," Applied Mathematics and Optimization, vol. 58, no. 1, pp. 29-67, 2008.

[32] A. Pazy, Semigroups of Linear Operators and Applications to Partial Differential Equations, Springer, New York, NY, USA, 1983.

[33] J. L. Lions, Quelques méthodes de résolution des problèmes aux limites non linéaires, Dunod, Paris, France, 1969. 


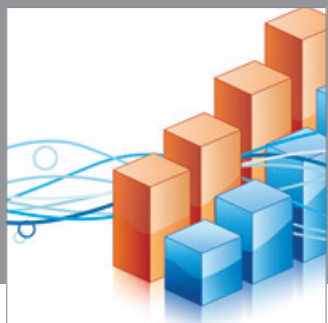

Advances in

Operations Research

mansans

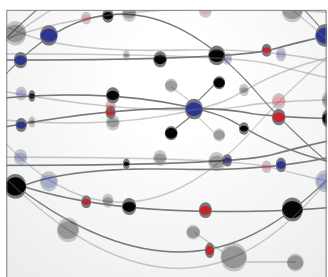

The Scientific World Journal
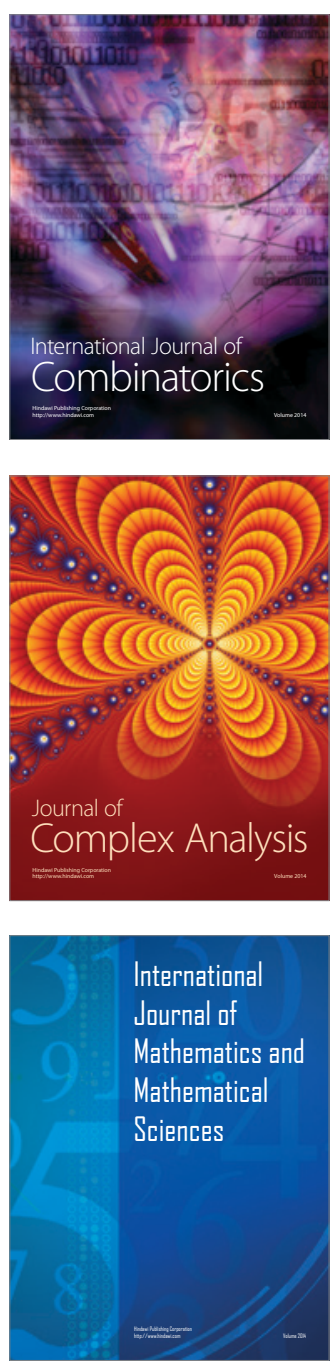
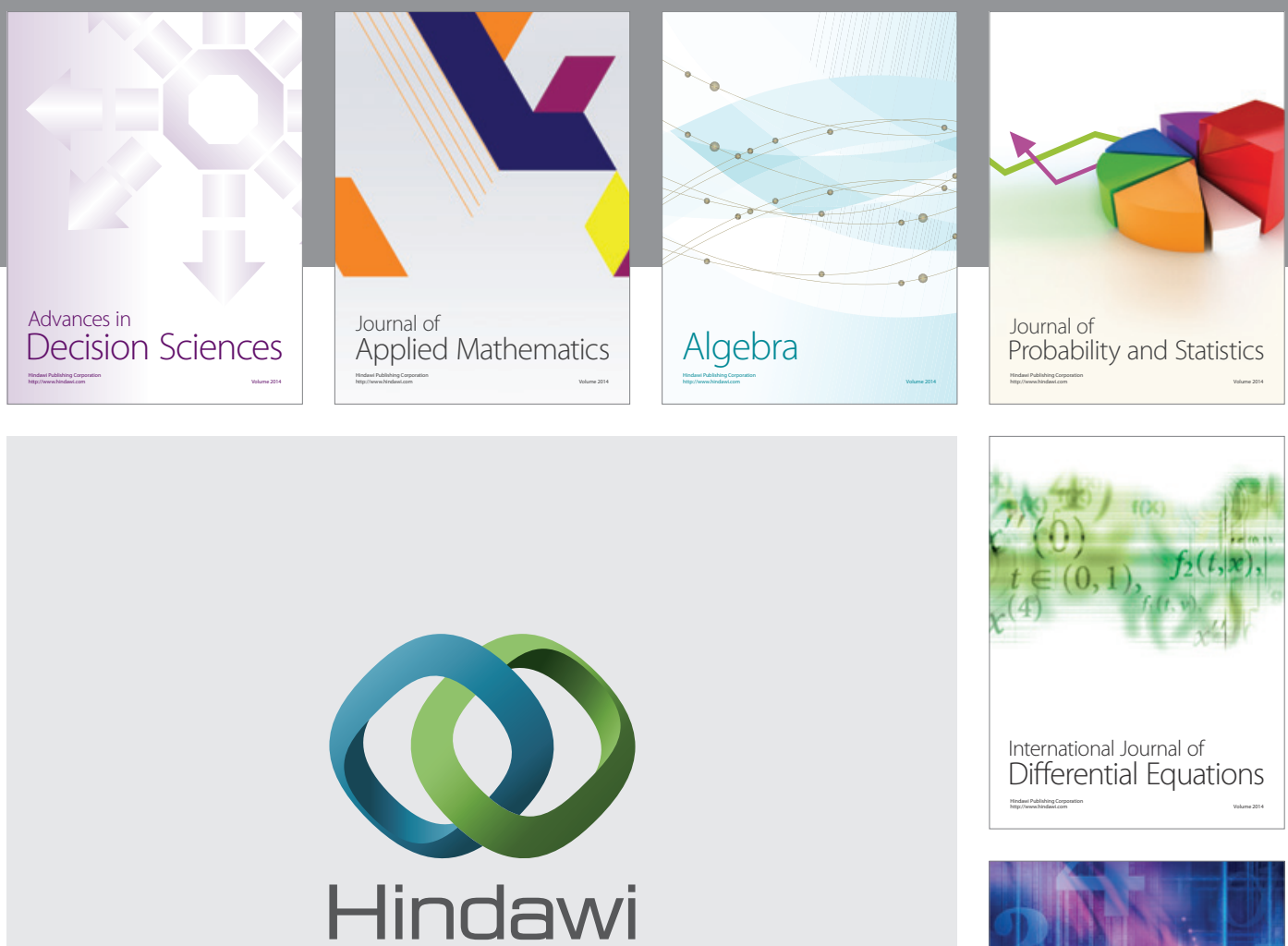

Submit your manuscripts at http://www.hindawi.com
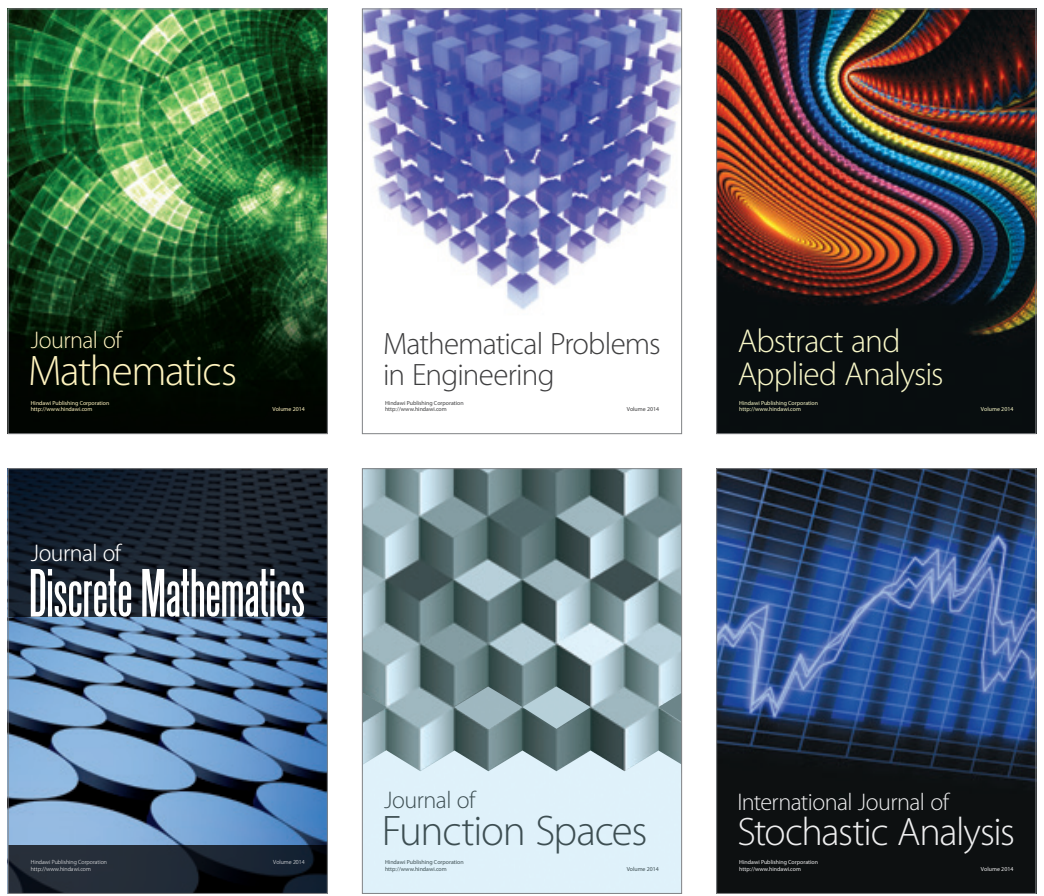

Journal of

Function Spaces

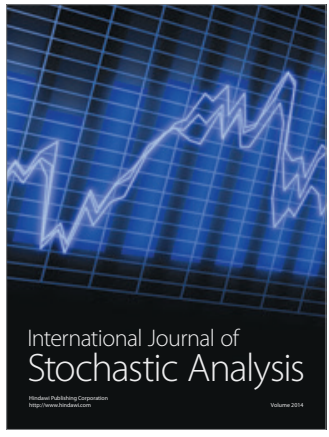

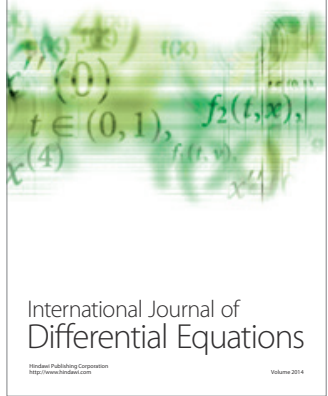
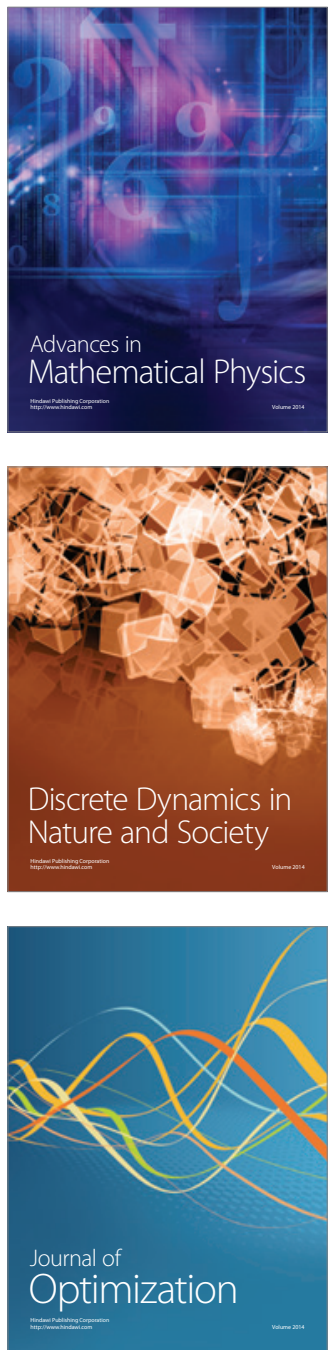